\title{
Nanoscale Confinement of Photo-Injected Electrons at Hybrid Interfaces SUPPORTING INFORMATION
}

\author{
Stefan Neppl ${ }^{1, \dot{+}, \dagger}$, Johannes Mahl ${ }^{1,2, \dagger}$, Friedrich Roth ${ }^{3}$, Giuseppe Mercurio ${ }^{2, \xi}$, Guosong Zeng ${ }^{1,4}$, \\ Francesca M. Toma ${ }^{1,4}$, Nils Huse ${ }^{2}$, Peter Feulner ${ }^{5}$, and Oliver Gessner ${ }^{l, *}$ \\ ${ }^{1}$ Chemical Sciences Division, Lawrence Berkeley National Laboratory, Berkeley, CA 94720, USA \\ ${ }^{2}$ Physics Department, Universität Hamburg and Center for Free-Electron Laser Science, 22761 Hamburg, Germany \\ ${ }^{3}$ Institute of Experimental Physics, TU Bergakademie Freiberg, 09599 Freiberg, Germany \\ ${ }^{4}$ Liquid Sunlight Alliance, Lawrence Berkeley National Laboratory, Berkeley, CA 94720, USA \\ ${ }^{5}$ Physics Department, Technische Universität München, 85748 Garching, Germany \\ these authors contributed equally to this work \\ *current affiliation: Paul Scherrer Institut, 5232 Villigen, Switzerland

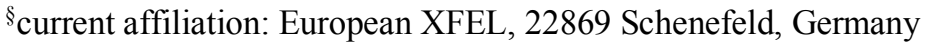

\section{Table of Contents}
A) Experimental details, sample preparation and characterization
B) Peak fitting analysis of the trXPS spectra
C) Ground state electronic structure of the $\mathrm{N} 3 / \mathrm{ZnO}$ interface
D) Control trXPS experiments on bare $\mathrm{ZnO}$ electrodes
E) Laser fluence dependence of trXPS effects
F) Retrieving potential gradients by numerical XPS peak simulations
G) Parallel plate capacitor model for interfacial potential drop
H) Rate equation model for 2-step charge injection and recombination
I) Time-resolved XPS of N3 adsorbed to a gold substrate
References

*email: ogessner@lbl.gov 


\section{A) Experimental details, sample preparation and characterization}

$\mathrm{ZnO}$ nanoparticles with an average diameter of $\sim 15 \mathrm{~nm}$ were prepared according to a procedure based on a zinc acetate dihydrate precursor (Sigma-Aldrich) in ethanoic solution. ${ }^{1}$ Approximately $0.2 \mathrm{ml}$ of the resulting colloidal suspension was spin-coated $(2,000 \mathrm{rpm})$ onto cleaned $2 \times 5 \mathrm{~cm}^{2}$ FTO glass slides. The films were subsequently dried at $60^{\circ} \mathrm{C}$ and sintered in air at $360{ }^{\circ} \mathrm{C}$ for 50 minutes. This procedure yields macroscopically homogenous nanoporous $\mathrm{ZnO}$ films (Fig. S1(a)), while preserving the nanoscale surface morphology (Fig. S1(b)). The direct bandgap of $E_{\mathrm{g}}$ $=3.30 \pm 0.01 \mathrm{eV}$ (see Fig. S1(c)) determined via the Tauc method ${ }^{2,3}$ is consistent with the literature. ${ }^{4}$ Sensitization with N3 dyes (cis-bis(isothiocyanato)bis(2,2'-bipyridyl-4,4'dicarboxylato)-ruthenium(II); Sigma-Aldrich) was carried out in ambient atmosphere by immersing the (still $\sim 80^{\circ} \mathrm{C}$ warm) $\mathrm{ZnO}$ films in a $0.2 \mathrm{mM}$ ethanoic solution of $\mathrm{N} 3$ for $3 \mathrm{~min}$. The resulting dye-sensitized $\mathrm{ZnO}$ films were thoroughly rinsed with ethanol to remove excess physisorbed molecules and blown dry in a stream of dry nitrogen. The comparably short sensitization time of $3 \mathrm{~min}$ was chosen to avoid the formation of $\mathrm{N} 3 / \mathrm{Zn}^{2+}$ surface complexes/aggregates, which exhibit inefficient electron injection properties and modify the surface morphology and electronic structure of the $\mathrm{ZnO}$ films due to dissolution of $\mathrm{Zn}^{2+}$ ions. ${ }^{5-8}$ While this precaution might be less important for bulk-sensitive measurements, such as all-optical studies, it is mandatory for the surface-sensitive trXPS experiments presented here. Samples were kept in the dark prior to transferring them into the vacuum chamber for the trXPS experiments carried out at a base pressure of $\sim 5 \cdot 10^{-8}$ mbar. We note that samples prepared using these or similar techniques have been shown to yield incident photon-to-current conversion efficiencies (IPCE) of $\sim 18 \%$ at $\sim 530 \mathrm{~nm}^{9}$

During the trXPS measurements, the sample was continuously scanned at a speed of $275 \mu \mathrm{m} / \mathrm{s}$, with a $500 \mu \mathrm{m}$ spacing between neighboring lines to exclude any modification of the ground-state (GS) electronic structure of the $\mathrm{N} 3 / \mathrm{ZnO}$ heterojunction due to $\mathrm{X}$-ray- and/or laser-induced damage. For the chosen experimental parameters, we do not observe any changes in the transient trXPS energy shifts or in the GS XPS line shapes and positions for two consecutive scans across the same sample area. We emphasize that fast sample scanning is imperative, since even exposure to synchrotron X-ray radiation itself can alter the GS surface-electronic structure of the $\mathrm{ZnO}$ electrodes, as illustrated for the Zn3d photoline in Fig. S1(d). Similar X-ray-induced effects have 
been observed in synchrotron-based XPS experiments on single crystalline $\mathrm{TiO}_{2}$ films. ${ }^{10}$ The $\mathrm{C} 1 \mathrm{~s}$ and Zn3d trXPS pump-probe data shown in Fig. 2(a) of the main text have been acquired in an interleaved fashion, i.e. acquiring $\mathrm{C} 1 \mathrm{~s}$ and $\mathrm{Zn3d}$ data at one pump-probe delay before moving to the next one. This procedure mitigates any possible influence of long-term experimental drifts on the quantitative comparison of the C1s and Zn3d trXPS transients in Fig. 2(a) of the main text. Xray photon energy changes required to switch between $\mathrm{C} 1 \mathrm{~s}$ and $\mathrm{Zn} 3 \mathrm{~d}$ regions do not affect the experiment, as an active feedback loop in the beamline stabilizes the X-ray beam pointing. Repeated tests of beam pointing fluctuations indicated a stability and reproducibility of $<5 \mu \mathrm{m}$ in horizontal and vertical directions for the two different X-ray energies. 


\section{B) Peak fitting analysis of the trXPS spectra}

\section{Fitting of the $C 1$ s/Ru3d spectral region}

The inset in Fig. 1 of the main text illustrates the molecular structure of the N3 dye. Carbon and ruthenium atoms are color-coded with corresponding colors used for the Gaussian peaks in Fig. 1(a) to indicate the different contributions to the C1s/Ru3d XPS spectrum. These peaks together with the sum of their Shirley backgrounds (dashed gray line) and a constant offset $(\sim 0.1)$ represent the result of a fit (blue line) of the GS spectrum (blue circles). In this fit, the Ru3d doublet (pink)

a

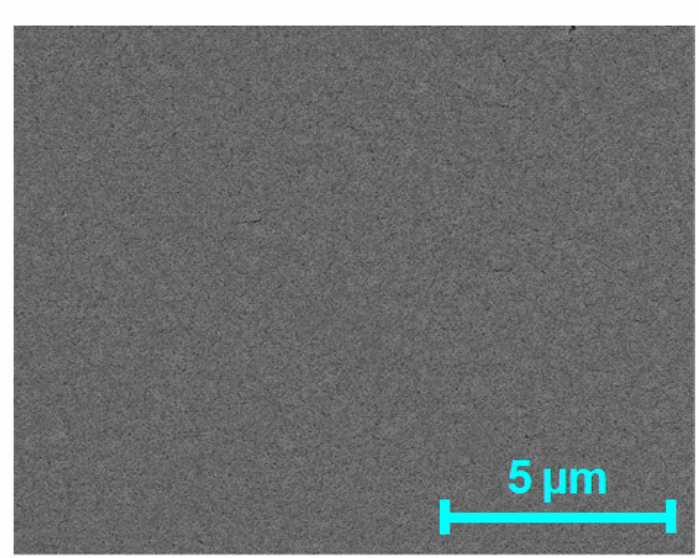

C

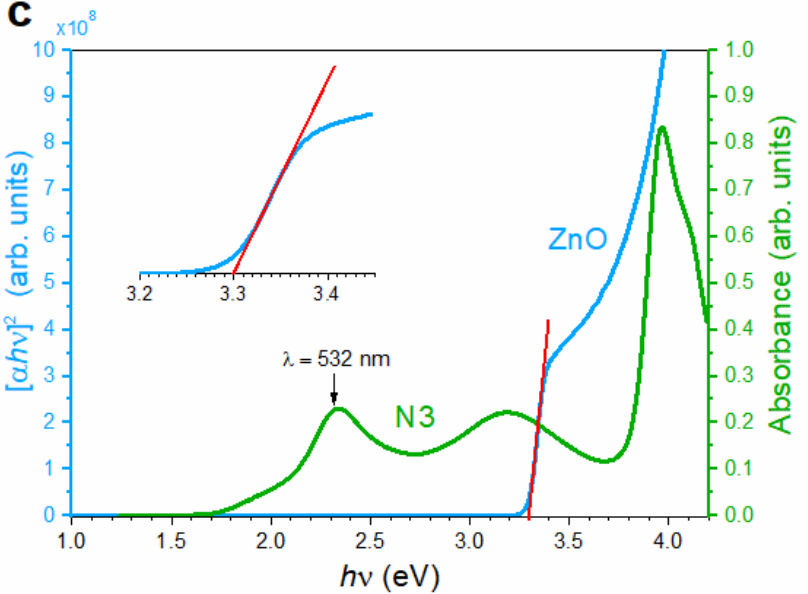

b

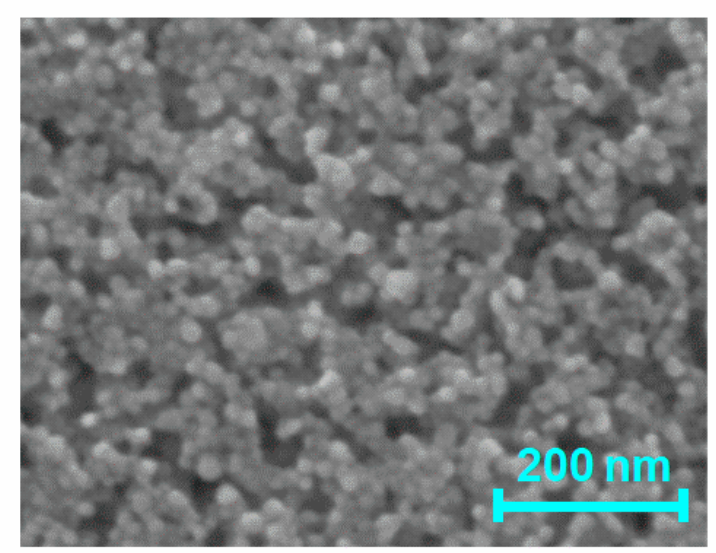

d

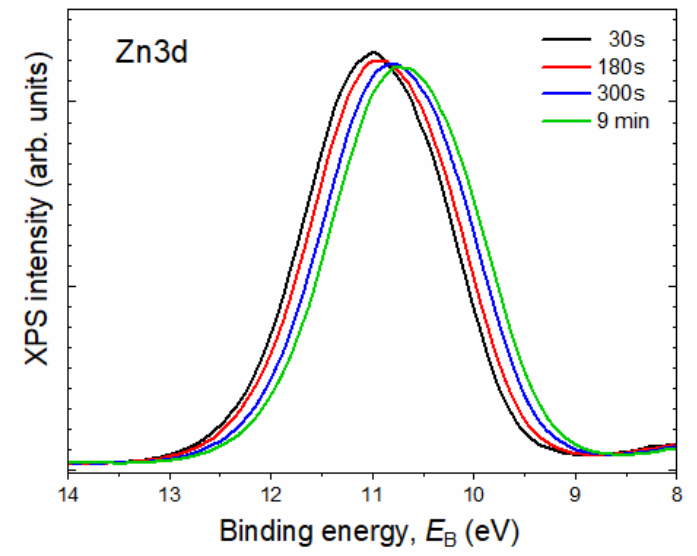

Figure S1. Characterization of ZnO nanoparticle substrates and N3 sensitizer. a,b SEM images of a $\mathrm{ZnO}$ nanoparticle film at different magnifications. $\mathbf{c}$ UV-Vis absorption spectra of $0.2 \mathrm{mM}$ $\mathrm{N} 3$ in ethanol (green) and the bare $\mathrm{ZnO}$ film (blue, scaled as indicated). The arrow indicates the pump wavelength $(532 \mathrm{~nm})$ used in the trXPS experiments. The inset highlights the determination of the direct bandgap via to the Tauc method (red line). d Zn3d XPS spectra at a fixed sample spot as a function of X-ray exposure time. 
has a fixed peak area ratio of $3 / 2$ between $R u 3 d_{5 / 2}$ and $R u 3 d_{3 / 2}$ and a fixed energy splitting of $4.2 \mathrm{eV} .{ }^{11}$ The Ru3 $\mathrm{d}_{5 / 2}$ peak appears at the lowest binding energy of $282.0 \mathrm{eV}$ and the less intense $\mathrm{Ru}_{3} \mathrm{~d}_{3 / 2}$ peak overlaps with the $\mathrm{C} 1 \mathrm{~s}$ signals from the bipyridyl rings at $285.5 \mathrm{eV}$ (blue, CCC) and $286.4 \mathrm{eV}$ (red, $\mathrm{CCN}$ ). The C1s signal from the thiocyanate groups (cyan, NCS) appears as a high binding energy shoulder $(287.6 \mathrm{eV})$ of the main feature, while the carbon in the carboxyl groups (green, $\mathrm{CCOOH}$ ) is subject to valence electron density depletion by the most electronegative environment, leading to the highest binding energy $\mathrm{C} 1 \mathrm{~s}$ component at $289.5 \mathrm{eV}$. For the fit procedure, the peak area ratios of the individual carbon peaks are fixed according to their respective number of occurrences within the molecule, i.e. 12:8:2:4 in the order CCC:CCN:NCS:CCOOH. Their relative energy separations are fixed to the values reported by Mayor et al, ${ }^{12}$ except for the carboxyl group, which is expected to have a substrate dependent binding energy. The spectral widths of the Ru3d doublet and the individual C1s components are free fit parameters, with the two bipyridine $\mathrm{C} 1$ s peaks constrained to have identical FWHM. ${ }^{12}$ For each data set, this GS fit is applied to the average of all data recorded at negative pump-probe delays. Compared to the GS spectrum, the only free parameters when fitting the ES spectra (red circles in Fig. 1(a)) are the relative binding energy shifts of the Ru3d doublet and the entire C1s emission, and the FWHM of the individual peaks as described for the GS. However, in contrast to the $\mathrm{Zn} 3 \mathrm{~d}$ data, no changes in the spectral widths between the GS and ES spectra are observed within the uncertainty of the fits. 


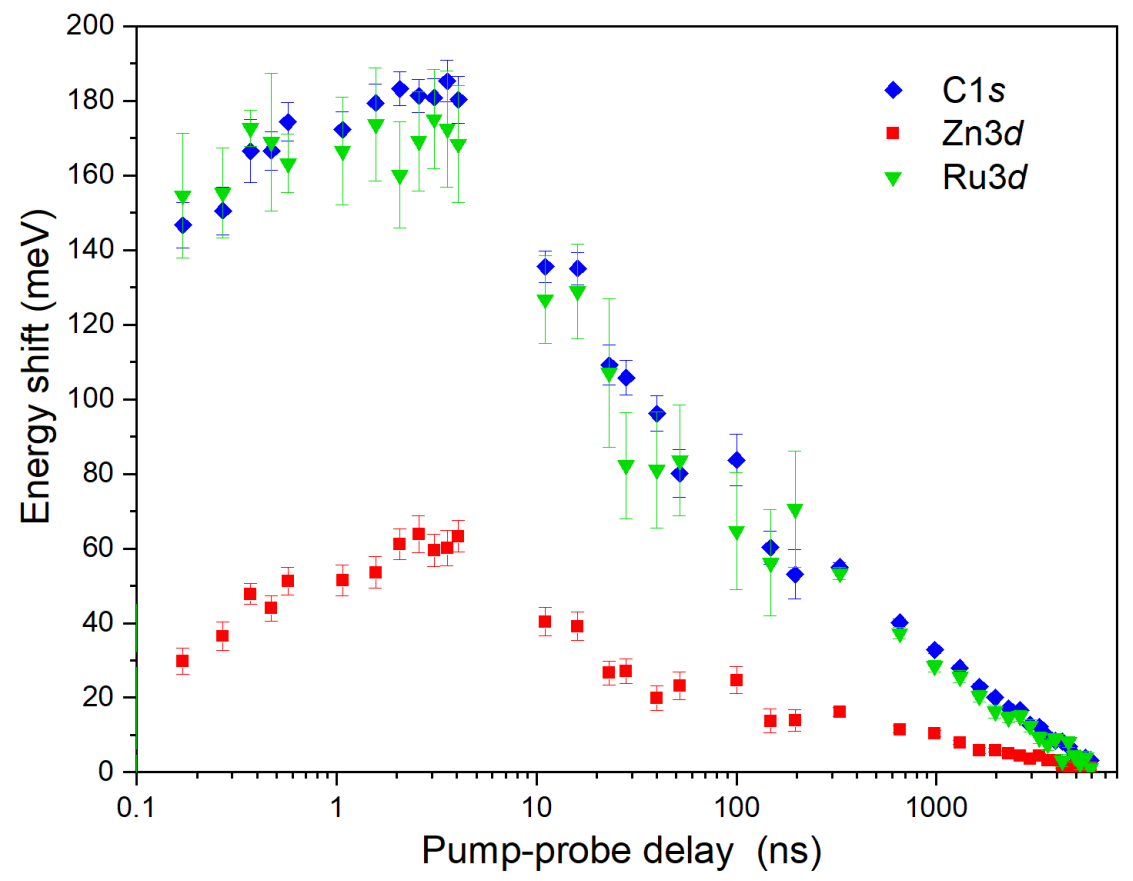

Figure S2. Spectral shifts of $\mathrm{C} 1 \mathrm{~s}, \mathrm{Ru} 3 \mathrm{~d}$, and $\mathrm{Zn3d}$ photolines as a function of pump-probe delay. The shifts of $\mathrm{C} 1 \mathrm{~s}$ (blue diamonds) and Ru3d (green triangles) are independent fit results. They agree within the uncertainty of the measurement, indicating a uniform shift of the entire $\mathrm{C} 1 \mathrm{~s} / \mathrm{Ru} 3 \mathrm{~d}$ spectral region associated with the N3 molecule. Zn3d trXPS shifts (red squares) from the $\mathrm{ZnO}$ semiconductor substrate are displayed for comparison.

Fig. S2 shows the resulting C1s (blue diamonds) and Ru3d (green triangles) shifts of the ES relative to the GS, which are identical within the experimental range of uncertainty. For improved clarity, only the C1s shifts are shown in Fig. 2(a) of the main text, as their determination is more precise.

\section{Fitting of the Zn3d spectral region}

The line-shape of the $\mathrm{Zn} 3 \mathrm{~d}$ emission is best described by an asymmetric Gaussian function. ${ }^{13}$ Fig. 1(b) of the main text shows the GS Zn3d spectrum (blue circles), which is fitted (blue line) with an asymmetric Gaussian peak on top of a polynomial background (gray dashed line). The free parameters of the asymmetric Gaussian peak fit are the peak area, the binding energy position of the maximum, a half-width-at-half-maximum (HWHM) value for the lower binding energy side, and an asymmetry factor $\alpha(>1)$ accounting for the broader peak shape on the higher binding energy side such that FWHM $=\operatorname{HWHM}(1+\alpha)$. The fit results are $\mathrm{FWHM}=1.53 \mathrm{eV}$ and $\alpha=1.18$ for $\mathrm{h} v=$ $320 \mathrm{eV}$, and $\mathrm{FWHM}=1.88 \mathrm{eV}$ and $\alpha=1.31$ for $\mathrm{h} v=614 \mathrm{eV}$. The difference in the $\mathrm{Zn} 3 \mathrm{~d}$ line-shape for the two data sets can be explained by the different energy resolution (beamline and analyzer) in the two experiments. 
As described for the $\mathrm{C} 1 \mathrm{~s} / \mathrm{Ru} 3 \mathrm{~d}$ spectrum, the GS fit for each $\mathrm{Zn} 3 \mathrm{~d}$ data set is performed on the average of all data with negative pump-probe delays. Each ES spectrum (red circles) is fitted (red line) individually with a variable binding energy position and FWHM parameter, while the peak area and asymmetry factor are fixed to the corresponding GS fit results. Resulting ES peak shifts with respect to the $\mathrm{GS}\left(\Delta \mathrm{E}_{\mathrm{B}}(\mathrm{Zn} 3 \mathrm{~d})\right)$, as well as the difference between ES and GS FWHM fit results $(\triangle \mathrm{FWHM}(\mathrm{Zn} 3 \mathrm{~d}))$ are shown in Fig. 2(a) of the main text. In addition to Fig. 1(b,d) in the manuscript, Fig. S3 provides a more comprehensive analysis of the photo-induced spectral changes of the Zn3d photoemission. Comparing the GS and ES spectra as recorded (Fig. S3(a)), with the same color coding as described above, both the shift and the broadening become apparent. Shifts to higher $E_{\mathrm{B}}$ are visible for both the low- and the high-energy side of the peak, highlighted in the right and bottom left insets, respectively. Furthermore, the ES peak broadening effect leads to a smaller amplitude of the ES spectrum compared to the GS, as seen in the top left inset in Fig. S3(a). Modelling the photo-induced effect by an $E_{\mathrm{B}}$ shift alone, as shown in Fig. S3(b), results in a distinct residual (bottom). However, a combination of $E_{\mathrm{B}}$ shift and spectral broadening of the GS spectrum (Fig. S3(c)) adequately describes the photo-induced effects in the ES spectrum, which is reflected in a significantly reduced residual. The amplitudes in this residual are similar to the fluctuations observed in difference spectra constructed from separate $\mathrm{Zn} 3 \mathrm{~d}$ spectra acquired with the same data acquisition time, but without any laser excitation. 

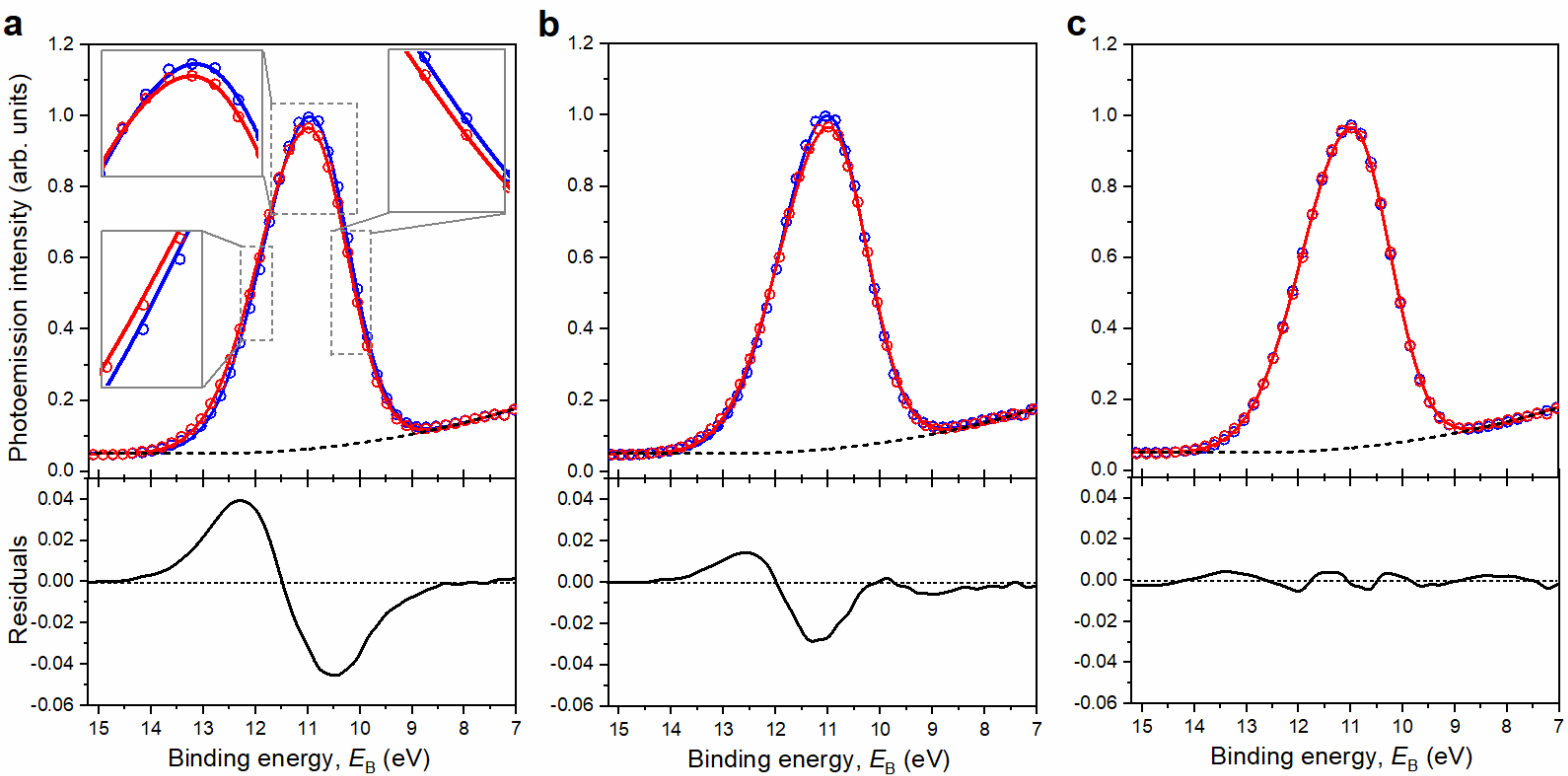

Figure S3. Photo-induced changes in Zn3d spectrum. a The data as recorded exhibit a shift towards higher binding energies in the ES (red) compared to the GS (blue) spectrum on both the high (bottom left inset) and low (right inset) binding energy sides of the Zn3d peak. Additionally, a photo-induced peak broadening leads to a decrease in the ES peak amplitude compared to the GS spectrum, visible in the top left inset. b A shift alone does not lead to satisfactory overlap of GS and ES spectra, as highlighted by the pronounced residual difference spectrum in the bottom panel. c Applying spectral broadening in combination with a binding energy shift to the GS spectrum leads to a good description of ES spectrum, and reduces the residual to a level comparable to the typical differences between two $\operatorname{trXPS}$ reference measurements without optical excitation. 


\section{C) Ground state electronic structure of the $\mathrm{N} 3 / \mathrm{ZnO}$ interface}

The GS band bending at the surface $V_{b b, \max }^{G S}$, and its spatial profile $V_{b b}^{G S}(z)$ for the hydrated, bare $\mathrm{ZnO}$ system has been established by temperature-resolved XPS/UPS experiments on the bare nanoporous $\mathrm{ZnO}$ film. ${ }^{14}$ In principle, the internal electric fields arising from local space-charge imbalances in the semiconductor surface region may be altered by the adsorption of a sensitizer. This would influence the energy-level alignment and coupling between the electron-donor and the semiconductor acceptor states. ${ }^{15-18}$ In the following, we demonstrate that N3 dye adsorption, when performed as described in Section A, does not change the GS band bending for the $\mathrm{N} 3 / \mathrm{ZnO}$ heterojunction compared to the hydrated, bare $\mathrm{ZnO}$ system.

Fig. S4 shows a series of XPS (Fig. S4(a,b)) and UPS (Fig. S4(c)) spectra of bare ZnO (upper panels) and $\mathrm{N} 3 / \mathrm{ZnO}$ films (lower panels). A non-monochromatized, laboratory based $\mathrm{MgK} \alpha \mathrm{X}$-ray source $(h v=1253.6 \mathrm{eV})$ is used for the XPS measurements, and the HeI emission from a UV lamp $(h v=21.2 \mathrm{eV})$ for the UPS characterization. Sample charging effects are excluded by reproducing
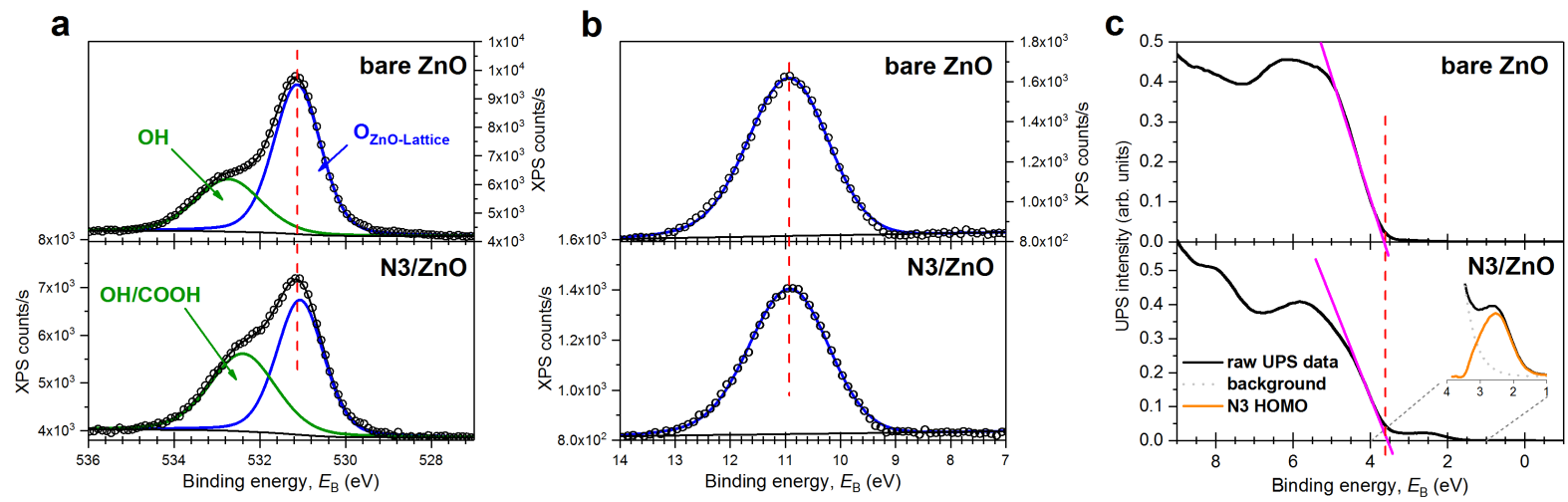

Figure S4. Static XPS and UPS characterization of bare (top) and N3-sensitized (bottom) nanoporous $\mathrm{ZnO}$ substrates. a O1s XPS spectra composed of ZnO-bulk (blue) and surfaceadsorbate (green) contributions. b Zn3d XPS photoemission associated with the $\mathrm{ZnO}$ electrode. c Determination of the VBM at the surface by linear extrapolation of the leading edge of the VB UPS emission (magenta). Top panels show the spectra of the hydrated bare $\mathrm{ZnO}$ electrode at room temperature. Spectra obtained from the N3-sensitized electrodes are depicted in the bottom panels. The red, vertical dashed lines highlight the alignment of features at relevant binding energies. The inset in (c) shows a magnified view of the N3 HOMO contribution to the $\mathrm{VB}$ emission for the sensitized substrate (orange) and the bare $\mathrm{ZnO}$ background (dashed gray). 
all spectra independent of the applied photon beam intensity, which was varied by more than one order of magnitude in both experiments. Additional tests were performed to rule out any impact of sample degradation due to prolonged X-ray/UV exposure.

The O1s spectra in Fig. S4(a) consist of a ZnO-lattice contribution (blue) and surface oxygen contributions (green) stemming from hydroxyl and, in the case of $\mathrm{N} 3 / \mathrm{ZnO}$, also carboxyl groups. In contrast, the Zn3d photoemission in Fig. S4(b) is exclusively associated with the semiconductor. For the analysis of the UPS spectra (Fig. S4(c)), a linear fit to the low binding energy edge (magenta line) is extrapolated to zero intensity, which yields the valence band maximum (VBM) position at the surface $E_{V B M}^{\text {surf }}$. Note that in the N3/ZnO heterojunction UPS data, there is an additional contribution from the N3 HOMO (orange peak) that does not affect the valence band position. Dashed red lines in all three panels demonstrate that the positions of the $\mathrm{ZnO}$-lattice associated O1s peak (Fig. S4(a)), the Zn3d peak (Fig. S4(b)) and $E_{V B M}^{\text {surf }}$ (Fig. S4(c)) are identical for the hydrated and $\mathrm{N} 3$-sensitized $\mathrm{ZnO}$ films within the accuracy of the fits. The binding energy axes are calibrated with respect to the Fermi level at $E_{\mathrm{F}}=0 \mathrm{eV}$ and the $A u 4 \mathrm{f}_{7 / 2}$ peak center at $84.00 \mathrm{eV}$ measured for a sputter-cleaned gold substrate with an accuracy of $\pm 10 \mathrm{meV}$. The identical peak and VBM energy positions for the bare and $\mathrm{N} 3$-sensitized $\mathrm{ZnO}$ substrates provide strong evidence that the amount of GS band bending is the same in both systems. ${ }^{18}$ Therefore, the amplitude and functional shape of $V_{b b}^{G S}(z)$ of the bare $\mathrm{ZnO}$ films are assumed to also represent the GS surface electronic structure of the $\mathrm{N} 3 / \mathrm{ZnO}$ heterojunction. 


\section{D) Control trXPS experiments on bare $\mathrm{ZnO}$ electrodes}

In order to exclude that photo-induced electron-hole pair generation within the $\mathrm{ZnO}$ electrode, e.g. via defect states within the bandgap, contribute to the observed photo-response, trXPS data on bare $\mathrm{ZnO}$ are acquired under the same experimental conditions as described in section A. Figure S5 compares $\mathrm{Zn3d}$ trXPS data acquired from the bare $\mathrm{ZnO}$ substrate (red squares) and the N3sensitized $\mathrm{ZnO}$ nanostructure (blue diamonds). Data for pump-probe delays larger than $100 \mathrm{ps}$ are shown in the main panel, and for near zero delays in the inset. No photo-response is detected for the bare $\mathrm{ZnO}$ sample. This result excludes significant contributions of electron-hole pairs generated within the $\mathrm{ZnO}$ substrate to the charge dynamics discussed in the main text.

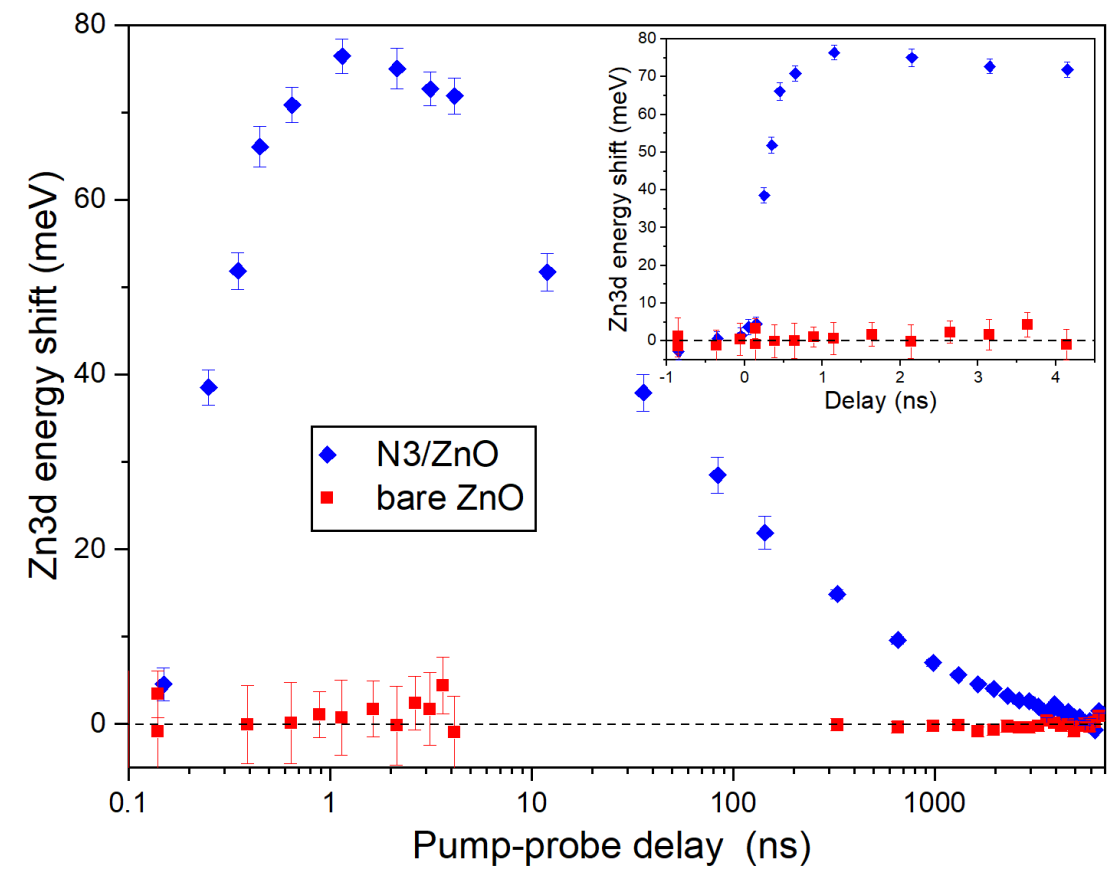

Figure S5. Control experiment with a bare $\mathrm{ZnO}$ substrate. Using identical pump laser conditions, the $\mathrm{Zn} 3 \mathrm{~d}$ spectrum of the $\mathrm{N} 3 / \mathrm{ZnO}$ heterojunction (blue diamonds) exhibits a significant photo-response, while the same line of the bare $\mathrm{ZnO}$ sample (red squares) is unaffected by the pump pulse. The inset shows a magnified view of data near zero pump-probe delay. 


\section{E) Laser fluence dependence of trXPS effects}

Fig. S6 shows the observed maximum shifts in the $\mathrm{C} 1 \mathrm{~s}$ (red) and Zn3d (blue) binding energies as a function of the optical pump pulse fluence. An approximately linear relationship at fluences up to $\sim 1 \mathrm{~mJ} / \mathrm{cm}^{2}$ is contrasted by a clear saturation effect toward higher fluences. The latter is likely, at least in part, caused by photodamage of the dye molecules, which is increasingly hard to control in the regime of $10 \mathrm{~s}$ of $\mathrm{mJ} / \mathrm{cm}^{2}$. Thus, experiments were performed at a fluence of $1.1 \mathrm{~mJ} / \mathrm{cm}^{2}$, which provides a compromise between a good signal/noise ratio in the trXPS data, and limited saturation/damage effects. Note that the observed trends provide strong evidence that nonlinear effects due to multiphoton absorption by the dye molecules do not contribute significantly to the observed dynamics.

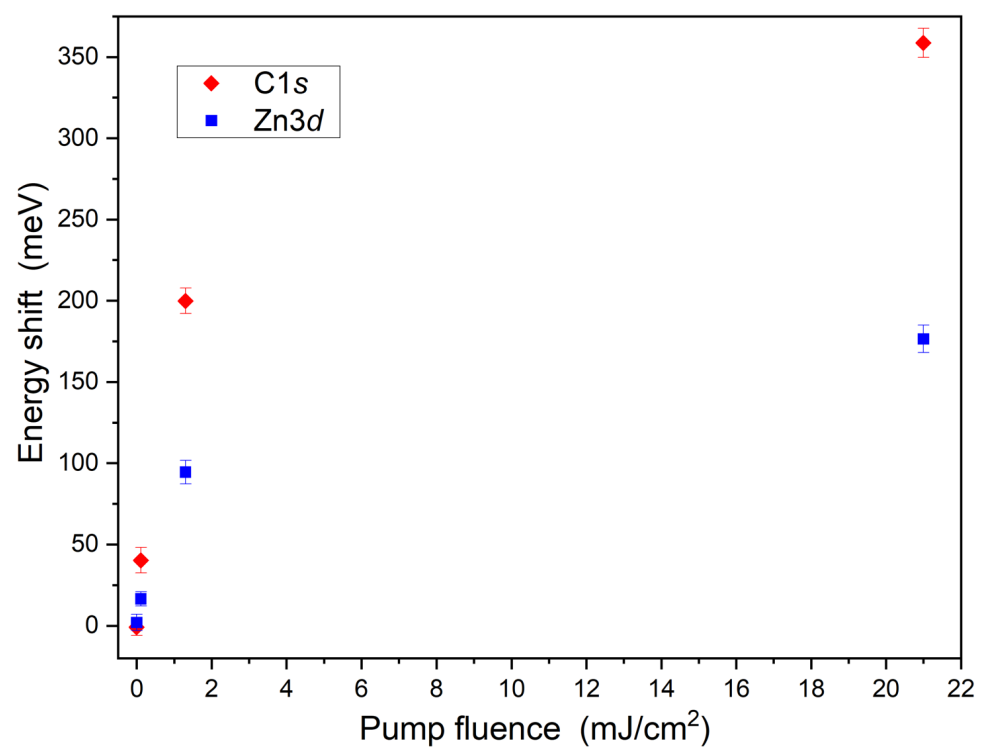

Figure S6. Pump pulse fluence dependence of the maximum energy shift in the $\mathrm{C} 1 \mathrm{~s}$ (red) and Zn3d (blue) photolines. 


\section{F) Retrieving potential gradients by numerical XPS peak simulations}

We apply an XPS peak reconstruction technique to retrieve information on the spatial profile of the internal potential $V_{b b}(z)$ from the Zn3d line-shape modifications. Similar methods have been successfully used in static XPS experiments to extract the spatial evolution of GS potential gradients at solid-liquid interfaces and at semiconductor heterojunctions from bias- and angledependent changes of XPS peak-shifts and broadening. ${ }^{19-25}$ The framework used to reconstruct the $\mathrm{Zn} 3 \mathrm{~d}$ photoemission peak for the bare $\mathrm{ZnO}$ film is described in detail in ref. 14. Briefly, the spectral distribution $D\left(E_{b}\right)$ of the $\mathrm{Zn} 3 \mathrm{~d}$ emission as a function of binding energy $E_{b}$ is simulated by a superposition of multiple photoemission lines with the same lineshape but different effective binding energies and amplitudes due to their emergence from different depths $z_{i}$ of the sample:

$$
\begin{aligned}
D\left(E_{b}\right) & =\int_{z \in S C R} D_{0}\left(E_{b}-E_{0}-V_{b b}(z)\right) e^{-\frac{z}{I M F P}} d z \\
& \approx \sum_{i(\forall z \in S C R)} D_{0}\left(E_{b}-E_{0}-V_{b b}\left(z_{i}\right)\right) e^{-z_{i} / I M F P}
\end{aligned}
$$

Here, $E_{0}$ denotes the binding energy at the peak maximum in the absence of any band bending, $D_{0}\left(E_{b}-E_{0}\right)$ the corresponding peak shape, $V_{b b}(z)$ the depth-dependent band bending, i.e. the deviation of the potential from the flat-band condition, as illustrated in Fig. 3 of the main text and Fig. S7 below, and IMFP the inelastic mean free path of photoelectrons in the $\mathrm{ZnO}$ substrate. The flat-band lineshape $D_{0}\left(E_{b}-E_{0}\right)$ is not known a priori. Instead, it is iteratively optimized in the simulations to reproduce the Zn3d photoemission spectra, using the GS FWHM and asymmetry parameters as starting values (see section B). The IMFP is calculated with the Tanuma-PowellPenn algorithm ${ }^{26}$ and determines the spectral weight $e^{-z_{i} / I M F P}$ of each individual contribution $D_{i}\left(E_{b}\right)=D_{0}\left(E_{b}-E_{0}-V_{b b}\left(z_{i}\right)\right)$ to the overall $\mathrm{Zn} 3 \mathrm{~d}$ photoemission signal. The spatial sampling step size $z_{i+1}-z_{i}$ is set to the average interlayer distance along the high-symmetry directions of the $\mathrm{ZnO}$ wurtzite lattice of $d_{L} \approx 0.2 \mathrm{~nm},{ }^{27}$ and the XPS sampling depth in the numerical simulation extends to 3 - IMFP. By comparing the peak shifts and FWHM changes of simulated Zn3d spectra $D\left(E_{b}\right)$ to experimental data as a function of pump-probe delay, the dynamic changes of the surface potential $V_{b b}(z)$ including the change at the surface $\Delta V_{b b}(z=0)$ can be accessed. The width of the space charge region (SCR), $d_{\mathrm{SCR}}$, describes the spatial extent across which $V_{b b}(z)$ values differ from the bulk value. Figure S7(b-d) schematically illustrates instructive cases of broadening of $D\left(E_{b}\right)$, or the lack thereof, depending on the relative length scales of $d_{\mathrm{SCR}}$ and IMFP. The largest 

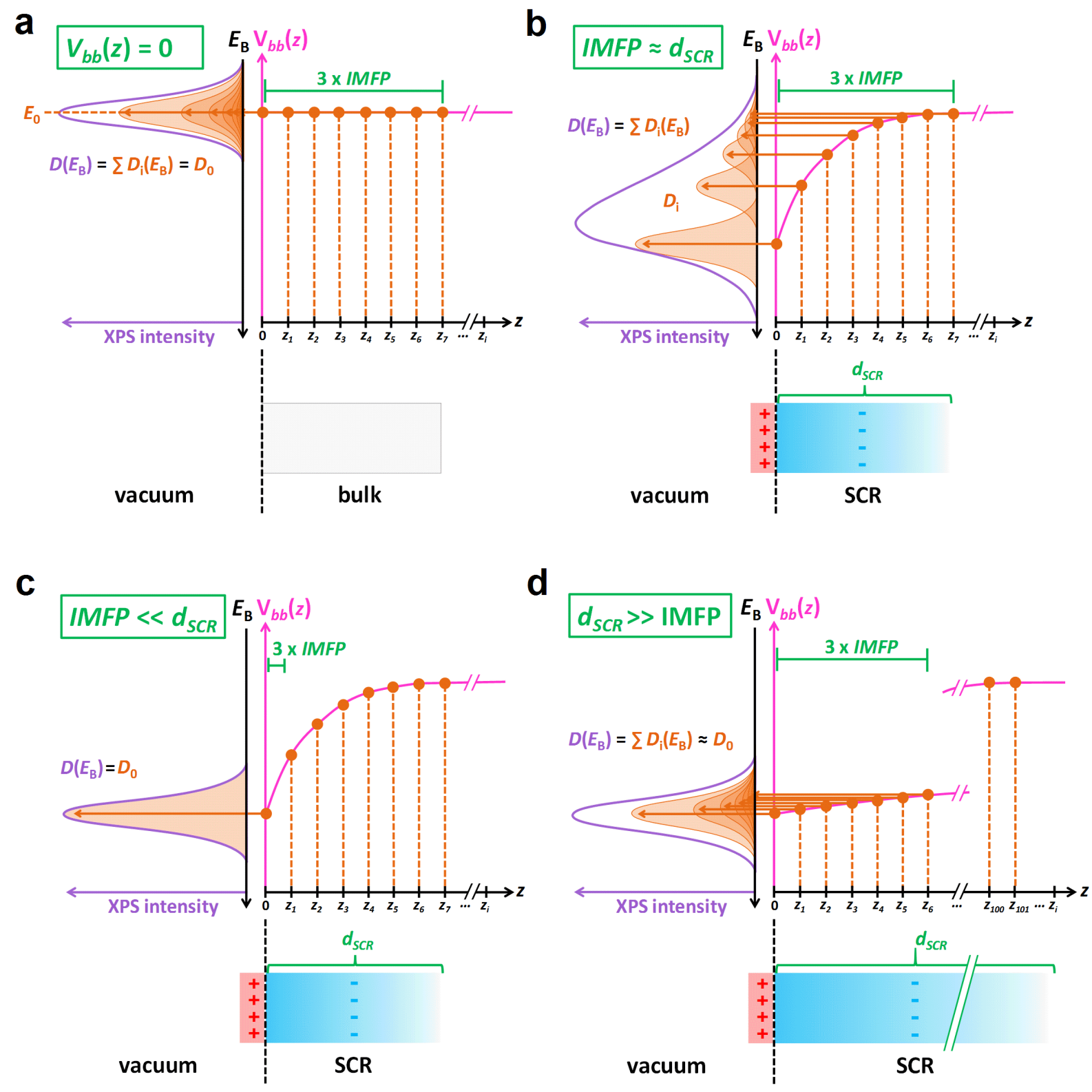

Figure S7. General correlation between the XPS lineshape $D\left(E_{b}\right)$, band-bending potential $V_{b b}(z)$ and $I M F P$. Different scenarios are schematically shown: a flatband conditions, $\mathbf{b}$ a narrow space-charge region (SCR) with downward band bending and $I M F P \approx d_{\mathrm{SCR}}, \mathbf{c}$ the same SCR but $I M F P<<d_{\mathrm{SCR}}$, and $\mathbf{d}$ a spatially very far extended SCL relative to IMFP. $D_{i}$ denotes the spectral photoemission contribution emerging from a distance $z_{i}$ from surface.

modulation of the XPS peak envelope occurs for IMFP $\approx d_{\mathrm{SCR}}$ (Fig. S7(b)), when the different contributions $D_{i}$ have markedly different binding energy positions and significant amplitudes. In this case, $D\left(E_{b}\right)$ will be noticeably broadened compared to flatband conditions. In all other cases illustrated in Fig. $\mathrm{S} 7, D\left(E_{b}\right) \approx D_{0}$ and no significant broadening will be observed. Specifically, 
small IMFPs (IMFP $\approx d_{L}<<d_{\mathrm{SCR}}$, see Fig. S7(c)), comparable with the interlayer lattice spacing $d_{L}$, lead to a suppression of contributions from deeper lying atoms and $D\left(E_{b}\right)$ consists only of contributions from the outermost surface without significant binding energy variations. In the other extreme case of a very extended SCR $\left(d_{\mathrm{SCR}}>>I M F P>>d_{L}\right.$, see Fig. S7(d)), deeper lying contributions have significant amplitude, but very similar binding energy positions due to the slowly varying internal semiconductor potential $V_{b b}(z)$. Hence, an approximate matching of IMFP and $d_{\mathrm{SCR}}$ is necessary to experimentally access $V_{b b}(z)$ through broadening effects in (tr)XPS data. This requirement highlights future prospects of trXPS in the tender/hard X-ray regime to probe light-induced modifications of potential gradients at hybrid systems that feature spatially more extended or buried SCRs compared to the prototypical N3/ZnO system explored here by soft Xray trXPS.

Fig. S8 displays the results of this envelope reconstruction approach for two exemplary GS spectra acquired at $h v=614 \mathrm{eV}$ (Fig. S8(a)) and $h v=320 \mathrm{eV}$ (Fig. S8(c)). For both spectra, the acquired experimental data (black dots) are described by $D\left(E_{b}\right)$, i.e., the SCR simulation (red line). Representative individual contributions $D_{i}$ at integer values of $z_{i} \leq 5 \mathrm{~nm}$ are shown as red shaded areas, where the sum of all $D_{i}$ defines $D\left(E_{b}\right)$ according to Eq. E1. The optimized $D_{0}$ flatband lineshape parameters are FWHM $=1.33 \mathrm{eV}$ and $\alpha=1.16$ at $h v=320 \mathrm{eV}$, and FWHM $=1.59 \mathrm{eV}$ and $\alpha$ $=1.35$ at $h v=614 \mathrm{eV}$. Figure S8(b,d) shows the depth-dependent relative peak positions, defined by $V_{b b}(z)$ (blue), and the respective weights derived from the IMFP (green). Parameters for $h v=614 \mathrm{eV}$ and $h v=320 \mathrm{eV}$ are shown in Fig. S8(b) and Fig. S8(d), respectively. The excellent agreement between the simulated and the measured GS XPS spectra in Fig. S8(a) and (c) demonstrates that a single, global surface potential $V_{b b}(z)$ together with one IMFP for each electron kinetic energy is sufficient to describe both experimental data sets with the envelope reconstruction approach.

We note that we have also evaluated alternative explanations for the observed photoinduced spectral changes, in particular, in the $\mathrm{Zn} 3 \mathrm{~d}$ line. Among these were an injection yield per excited dye molecule of less than 1 as well as the possibility of spatially inhomogeneous charge injection that, in principle, may lead to separate regions with and without band banding effects. None of these alternatives to the model presented above provides a satisfactory, self-consistent explanation 
a

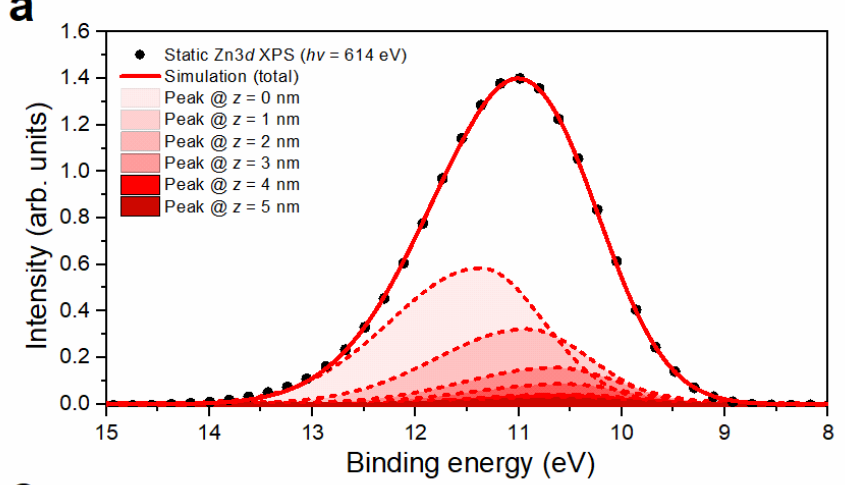

\section{C}

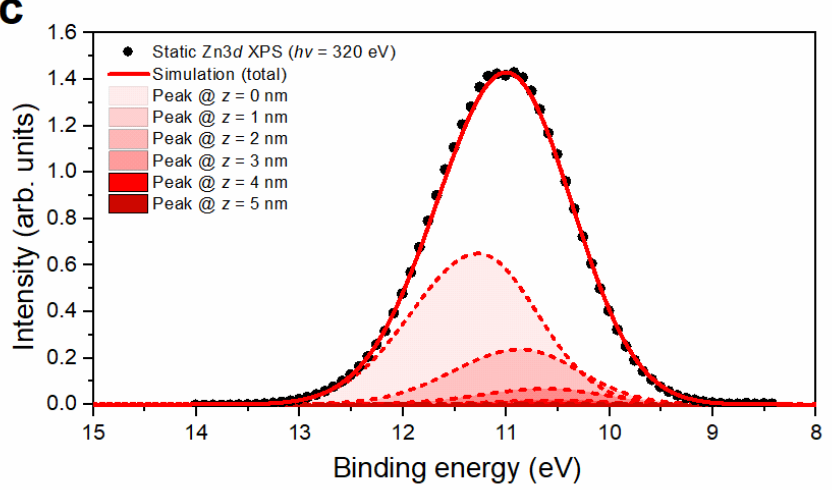

b

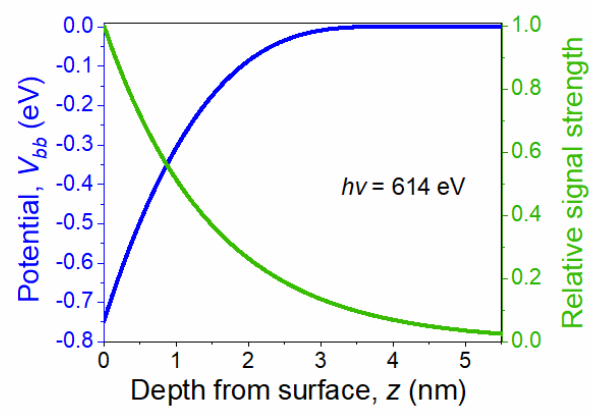

d

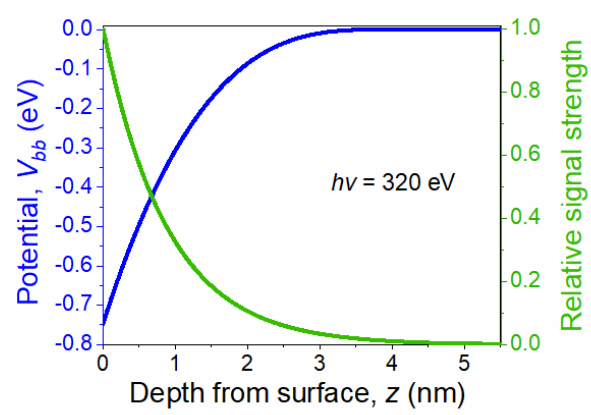

Figure S8. Comparison between measured GS Zn3d spectra and a numerical SCR simulation. a Data (black dots) acquired at $h v=614 \mathrm{eV}$ and corresponding SCR simulation (red line). Representative contributions $D_{\mathrm{i}}$ from different depths $z_{i}$ are shown as red-shaded peaks. b Near surface potential $V_{\mathrm{bb}}$ (blue) and relative XPS signal contributions (green) as a function of depth $z$, which determine the energy position $\mathrm{E}_{\mathrm{B}}$ and weight of $D_{\mathrm{i}}$, respectively. c Data and simulation results for $h v=320 \mathrm{eV}$. d Depth-dependent potential $V_{\mathrm{bb}}$ (blue) and XPS signal contributions (green) that underlie the simulation shown in panel (c).

of the data. For example, a spatially inhomogeneous injection has been approximated by a model with two free fit parameters for the $\mathrm{Zn} 3 \mathrm{~d}$ line, representing the fraction of regions with injection and the injecting regions' spectral shift. This model did not yield satisfactory fit results. Furthermore, if there were regions of excited dye molecules that do not inject, one would expect fractional line-shape changes in the region of the $\mathrm{C} 1 \mathrm{~s} / \mathrm{Ru} 3 \mathrm{~d}$ spectrum since signals from dye molecules in injecting regions would very likely be affected differently than those in non-injecting regions. However, careful analysis of these signals did not reveal any evidence for such effects, but instead the entire $\mathrm{C} 1 \mathrm{~s} / \mathrm{Ru} 3 \mathrm{~d}$ undergoes a rigid shift as illustrated in Fig. 1 of the main manuscript. We also emphasize again that we carefully avoided possible complications due to high dye loading by limiting the substrate sensitization time to only 3 minutes, as described in section A. 


\section{G) Parallel plate capacitor model for interfacial potential drop}

The injected electron in the $\mathrm{ZnO} \mathrm{CB}$ and the hole remaining in the $\mathrm{HOMO}$ of the $\mathrm{N} 3$ dye molecule can be viewed as a photo-induced transient dipole that manifests itself as an interfacial potential drop (IPD) within the $\mathrm{N} 3 / \mathrm{ZnO}$ system. In a first order description, the injected electron can be assumed to experience metal-like, perfect screening within the $\mathrm{ZnO}$. Within this approximation, the interfacial potential drops between the $\mathrm{HOMO}$ hole and the $\mathrm{ZnO}$ surface, and is not sensitive to the exact position of the injected electron within the substrate. Experimentally, the IPD is accessible as $\Delta E_{\mathrm{N} 3}$, i.e. the difference between the $\mathrm{C} 1 \mathrm{~s} / \mathrm{Ru} 3 \mathrm{~d}$ shifts, displayed in Fig. 2(a) of the main manuscript, and the $\mathrm{Zn3d}$ shift at the surface, i.e. $\Delta V_{b b}(z=0)$. It is important to realize that the detected kinetic energy of $\mathrm{Zn} 3 \mathrm{~d}$ photoelectrons is not affected by the IPD, despite their passage through it on their way to the detector. The detector and $\mathrm{ZnO}$ sample are both grounded and, therefore, share a common Fermi energy level. Hence, the detected kinetic energy of photoelectrons originating from the $\mathrm{ZnO}$ substrate is a function of photoelectron binding energy, $\mathrm{X}$-ray photon energy and detector work function only. See e.g. Fig. 2 in ref. 28 for a more detailed explanation. However, photoelectrons emitted by an adsorbate are expected to be affected by the (transient) IPD. ${ }^{18,29}$ Similar to static energy shifts induced by interfacial electric fields or surface dipoles as described by Lueth, ${ }^{30}$ the IPD can be modeled as a voltage drop across a parallel plate capacitor. Here, the dye HOMO holes and the $\mathrm{ZnO}$ surface each constitute one side of the capacitor. For this purpose, the nanoporous structure of the sample is assumed to be approximately described by a (locally) flat surface. Within this approximation, the electric field associated with the IPD is described by $E=\Delta E_{N 3} / d_{I P D}$, where $d_{I P D}$ is the distance between the idealized plates, i.e., between $\mathrm{HOMO}$ holes and $\mathrm{ZnO}$ surface. Using the general relation $C=Q / \Delta E_{N 3}$ between the stored charge $Q$, the voltage drop $\Delta E_{N 3}$, and the capacitance $C$, as well as the parallel-plate capacitor relation $C=\varepsilon_{0} \varepsilon_{r} A / d_{I P D}$, with the vacuum permittivity $\varepsilon_{0}$, the relative permittivity of the medium between the two plates $\varepsilon_{r}$, and the area of each individual plate $A$, and taking into account the interaction of adjacent dipoles, leads to: ${ }^{28}$

$$
d_{I P D}=\left(1+f_{\text {dep }}\right) \Delta E_{N 3} \varepsilon_{0} \varepsilon_{r} A / Q=\left(1+f_{\text {dep }}\right) \Delta E_{N 3} \varepsilon_{0} \varepsilon_{r} / \rho
$$

Here, $\rho$ is the surface charge density $\rho=Q / A$, and the factor $1+f_{\text {dep }}$ is a correction that accounts for the mutual dipole interaction. The so-called depolarization factor $f_{d e p}$ for a square array of uniformly arranged charge pairs is given by $f_{d e p} \cong 9 \alpha \rho^{3 / 2} / 4 \pi \varepsilon_{0},{ }^{30}$ with $\alpha$ being the polarizability 
of the adsorbed molecules. N719, which is very similar to the N3 dye, has a calculated polarizability of $1.06 \cdot 10^{-1} \mathrm{~nm}^{3} .{ }^{31}$ The surface charge density $\rho$ is estimated by

$$
\rho=P_{e x} P_{\text {in }} \rho_{M}
$$

where $P_{e x}$ is the molecule excitation probability, $P_{i n}$ is the electron injection probability for an excited molecule, and $\rho_{M}$ is the number of adsorbed molecules per surface area. Values for $\rho_{M}$ vary between $\sim 0.5$ and $\sim 1.0 \mathrm{~nm}^{-2}$, based on experimental $\left(0.55 \mathrm{~nm}^{-2}\right)^{32}$ and theoretical $\left(0.77-1.16 \mathrm{~nm}^{-2}\right)^{33}$ studies. $P_{\text {in }}$ of excited N3 molecules attached to a nanocrystalline film of $\mathrm{ZnO}$ has been estimated as being close to unity based on transient optical absorption spectroscopy. 6,34 $P_{e x}$ is defined as

$$
P_{e x}=\sigma_{N 3}(532 \mathrm{~nm}) N_{p h}
$$

where $\sigma_{N 3}(532 \mathrm{~nm}) \approx 20$ Mbarn is the cross-section of N3 molecules for excitation with $532 \mathrm{~nm}$ light, ${ }^{35}$ and $N_{p h}=2.95 \cdot 10^{15} \mathrm{~cm}^{-2}$ is the number of photons per laser pulse per unit area derived from the laser fluence of $1.1 \mathrm{~mJ} \mathrm{~cm}^{-2}$. This leads to a fraction of excited molecules $P_{e x}=5.9 \%$ and a surface charge density $\rho=5.9 \cdot 10^{-2} e \cdot \mathrm{nm}^{-2}$ (for $\rho_{M}=1.0 \mathrm{~nm}^{-2}$ ). For this surface charge density, the effect of the depolarization factor $f_{d e p}$ is relatively small at $1.4 \%$. The relative permittivity $\varepsilon_{r}$ of a (mono-)layer of molecules is not necessarily related to a macroscopic or bulk-like property. ${ }^{36}$ Using values of $\varepsilon_{r} \approx 4-8,{ }^{36-40} \rho_{M} \approx 0.5-1.0 \mathrm{~nm}^{-2}$, and $\Delta E_{\mathrm{N} 3}=80 \mathrm{meV}$, Eq. F1 leads to a distance between $\mathrm{HOMO}$ hole and $\mathrm{ZnO}$ surface of $0.3-1.2 \mathrm{~nm}$. As noted in the manuscript, these distances are compatible with the height of $\sim 1.0-1.3 \mathrm{~nm}$ of $\mathrm{N} 3$ dyes anchored to $\mathrm{TiO}_{2}$ substrates, ${ }^{41}$ and the fact that the HOMO holes are primarily located on the Ru centers and NCS groups of the dye molecules. $^{42}$

Within the parallel plate model, the above parameters correspond to an average electric field of $\sim 66$ to $260 \mathrm{MV} / \mathrm{m}$ within the IPD. Interfacial electric fields in dye-sensitized systems have previously been investigated via the Stark effect in optical absorption spectra. ${ }^{43-45}$ Time-resolved Stark effect optical absorption studies found interfacial fields of $35 \mathrm{MV} / \mathrm{m}^{44}$ and $270 \mathrm{MV} / \mathrm{m}^{45}$ resulting from photoexcited electron-hole pairs at different ruthenium based sensitizers on $\mathrm{TiO}_{2}$ in the presence of an electrolyte. The results presented here demonstrate that trXPS provides a complementary route to gain information on interfacial electric fields as well as the spatial distance of transient charge carrier densities relative to the electrode surface. 


\section{H) Rate equation model for 2-step charge injection and recombination}

Figure S9(a) illustrates the coupled rate equation model used to describe the time-dependent N3 HOMO hole population $P_{\mathrm{H}}(\mathrm{t})$, as well as the electron populations $P_{\mathrm{LUMO}}(t), P_{\mathrm{ICT}}(t)$ and $P_{\mathrm{CB}}(t)$ in the N3 LUMO, in interfacial charge transfer (ICT) states at the surface, and in the $\mathrm{ZnO}$ conduction band (CB), respectively. The model is based on the first-order approximation that the timedependent change of any given electron population $P_{i}$ is given by the difference between the rate of growth from electron-donating channels and the rate of electron loss into other electron acceptors, whereby the rates are characterized by time-independent rate constants:

$$
\frac{d}{d t} P_{i}(t)=\sum_{j} k_{j}^{I N} P_{j}^{I N}(t)-\sum_{m} k_{m}^{\text {OUT }} P_{i}(t)
$$

Here, $P_{j}^{I N}(t)$ are the time-dependent populations that donate electrons to $P_{i}$ at rates $k_{j}^{I N} P_{j}^{I N}(t)$, and $k_{m}^{\text {OUT }}$ are the time-independent rate constants for the loss of electrons from $P_{i}$ into other electron acceptors.

The N3 LUMO is populated essentially instantaneously from the HOMO by the optical laser pulse $\left(k_{1}\right)$ and constitutes the first relevant population. The change of $P_{\text {LUMO }}$ is proportional to the rate of electron transfer to the surface $\left(k_{2}\right)$ and the recombination rate between the HOMO hole and the LUMO electron $\left(k_{1 R}\right)$ :

$$
\frac{d}{d t} P_{L U M O}(t)=-\left(k_{2}+k_{1 R}\right) P_{L U M O}(t)
$$

leading to

$$
P_{L U M O}(t)=P_{0} \exp \left(-\left[k_{2}+k_{1 R}\right] t\right),
$$

with $P_{\text {LUMO }}(t=0)=P_{0}$ as the initial LUMO population created by the pump laser. The corresponding rate equation for $P_{I C T}(t)$ is

$$
\frac{d}{d t} P_{I C T}(t)=k_{2} P_{L U M O}(t)-\left(k_{3}+k_{4}\right) P_{I C T}(t) .
$$




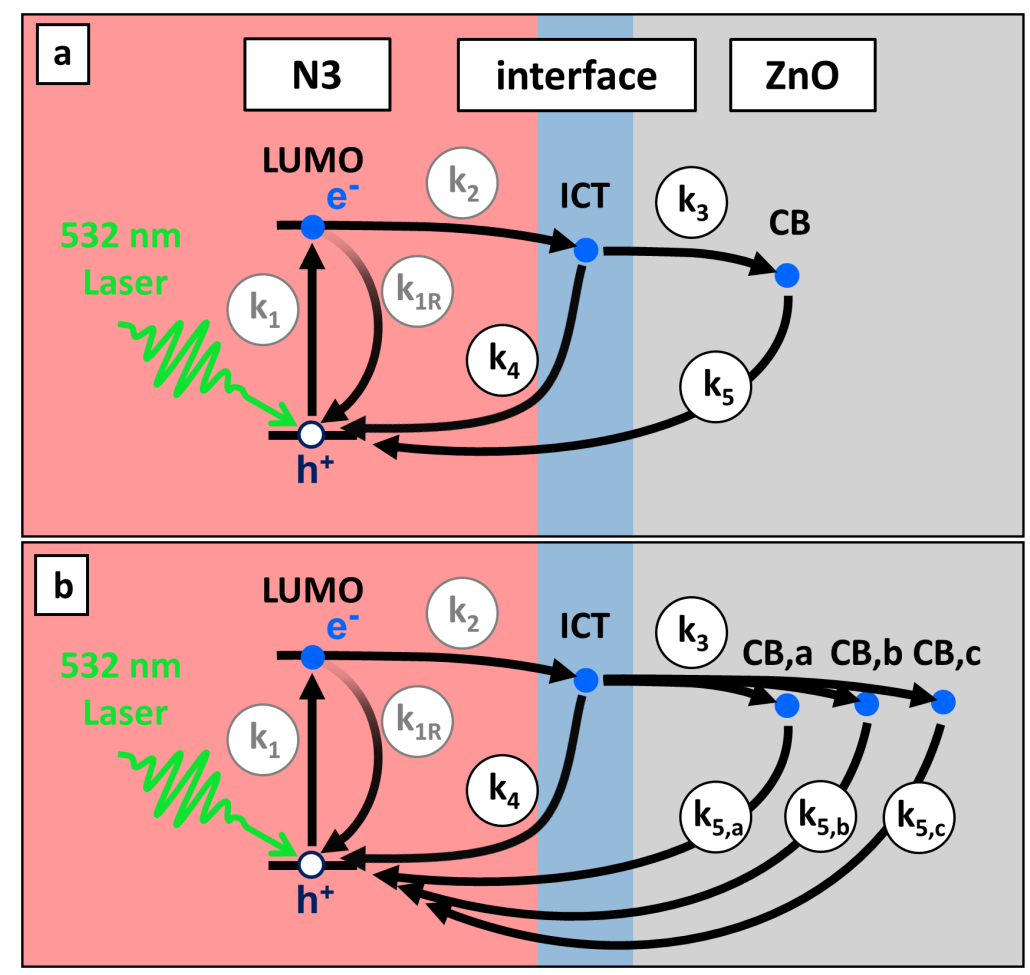

Figure S9. Coupled rate equation model describing photo-induced charge separation, injection and recombination in $\mathrm{N} 3-\mathrm{ZnO}$ heterojunction. a Laser light $(532 \mathrm{~nm}$, green) is absorbed by N3 dye molecules (red background), leading to a HOMO-LUMO transition $\left(k_{1}\right)$. The electron in the LUMO (e) either proceeds to the interface $\left(k_{2}\right)$ to form an interfacial charge transfer (ICT) configuration, or it recombines $\left(k_{1 \mathrm{R}}\right)$ with the hole $\left(\mathrm{h}^{+}\right)$. From the ICT configuration, electron injected into the $\mathrm{ZnO}$ conduction band $(\mathrm{CB})\left(k_{3}\right)$ is again competing with recombination $\left(k_{4}\right)$. Finally, electrons injected into the $\mathrm{ZnO}$ electrode $\mathrm{CB}$ recombine with holes in the surface-bound dye molecules with a characteristic rate $k_{5}$. This effectively describes a mono-exponential decay behavior from the $\mathrm{ZnO} \mathrm{CB}$. b A tri-exponential decay can be implemented via three distinct pathways $\mathrm{a}, \mathrm{b}$, and $\mathrm{c}$ that receive the same total number of electrons with the total rate constant $k_{3}$ as in panel (a), while the ratio of the number of electrons per pathway and their decay rates $\left(k_{5, \mathrm{a}}, k_{5, \mathrm{~b}}, k_{5, \mathrm{c}}\right)$ are different. See text for details. The rates $\mathrm{k}_{1}, \mathrm{k}_{2}$, and $\mathrm{k}_{1 \mathrm{R}}$ (gray) are not accessible in the experiment and taken from the literature. Rates $\mathrm{k}_{3}, \mathrm{k}_{4}$, and $\mathrm{k}_{5 \mathrm{a}}, \mathrm{k}_{5 \mathrm{~b}}, \mathrm{k}_{5 \mathrm{c}}$ (black) are free fit parameters.

Here, $k_{3}$ is the rate of electron injection into the $\mathrm{CB}$ from ICT states and $k_{4}$ is the recombination rate of ICT electrons with N3 HOMO holes. To solve this equation, we can use Eq. G3 and multiply by $\exp \left(\left[k_{3}+k_{4}\right] t\right)$, after which integration by parts, and the fact that there are no charge carriers at the surface at the moment of excitation, i.e. $P_{I C T}(t=0)=0$, leads to

$$
P_{I C T}(t)=\frac{k_{2} P_{0}}{k_{3}+k_{4}-\left\{k_{2}+k_{1 R}\right\}}\left[\exp \left(-\left[k_{2}+k_{1 R}\right] t\right)-\exp \left(-\left[k_{3}+k_{4}\right] t\right)\right] .
$$


Finally, electrons from the surface-located ICT states are injected into the $\mathrm{ZnO} \mathrm{CB}$ with a rate of $k_{3}$ and recombine from the $\mathrm{CB}$ with $\mathrm{N} 3 \mathrm{HOMO}$ holes at a rate of $k_{5}$ (Fig. S9(a)), leading to

$$
\frac{d}{d t} P_{C B}(t)=k_{3} P_{I C T}(t)-k_{5} P_{C B}(t) .
$$

This equation is solved in a similar fashion as shown above for $P_{I C T}(t)$ and, together with Eq. $\mathrm{G} 5$, yields the time-dependent electron population in the $\mathrm{ZnO} \mathrm{CB}$ :

$$
P_{C B}(\mathrm{t})=\frac{k_{3} k_{2} P_{0}}{k_{3}+k_{4}-\left\{k_{2}+k_{1 R}\right\}}\left\{\frac{\exp \left(-\left[k_{2}+k_{1 R}\right] t\right)-\exp \left(-k_{5} t\right)}{k_{5}-k_{2}-k_{1 R}}-\frac{\exp \left(-\left[k_{3}+k_{4}\right] t\right)-\exp \left(-k_{5} t\right)}{k_{5}-k_{3}-k_{4}}\right\} .
$$

This solution for $P_{C B}(\mathrm{t})$ effectively incorporates a mono-exponential decay of the $\mathrm{ZnO} \mathrm{CB}$ population with one rate constant, as shown in Fig. S9(a). The distinctly different timescales for injection and recombination within the system manifest themselves as a plateau for both the $\mathrm{C} 1 \mathrm{~s}$ and $\mathrm{Zn3d}$ trXPS data in Fig. 2(a) of the main text. This difference in timescales enables a simple, straightforward inclusion of the observed multi-exponential decay behavior of the $\mathrm{CB}$ electron population in the model.

The trXPS data are best described by a tri-exponential fit. Therefore, as depicted in Fig. S9(b), three different pathways a, b, and c are introduced with independent recombination rates $k_{5, \mathrm{a}}, k_{5, \mathrm{~b}}$, and $k_{5, \mathrm{c}}$, respectively. These pathways share the electron population provided by the ICT state decay ( $\left.\mathrm{k}_{3}\right)$, using individual amplitude ratios $R_{C B, a}, R_{C B, b}$, and $R_{C B, c}$ with respect to the overall electron population, such that

$$
\boldsymbol{R}_{C B, a}+\boldsymbol{R}_{C B, b}+\boldsymbol{R}_{C B, c}=1
$$

The resulting populations $P_{C B, a}(\mathrm{t}), P_{C B, b}(\mathrm{t})$, and $P_{C B, c}(\mathrm{t})$ are identical to Eq. $\mathrm{G} 7$ except for individual ratios $R_{C B, i}$ as pre-factors and recombination rates $k_{5, \text { i }}$ instead of $k_{5}$ such that

$$
P_{C B, a}(\mathrm{t})=\frac{R_{C B, a} k_{3} k_{2} P_{0}}{k_{3}+k_{4}-\left\{k_{2}+k_{1 R}\right\}}\left\{\frac{\exp \left(-\left[k_{2}+k_{1 R}\right] t\right)-\exp \left(-k_{5, a} t\right)}{k_{5, a}-k_{2}-k_{1 R}}-\frac{\exp \left(-\left[k_{3}+k_{4}\right] t\right)-\exp \left(-k_{5, a} t\right)}{k_{5, a}-k_{3}-k_{4}}\right\}
$$

and

$$
P_{C B, b}(\mathrm{t})=\frac{R_{C B, b} k_{3} k_{2} P_{0}}{k_{3}+k_{4}-\left\{k_{2}+k_{1 R}\right\}}\left\{\frac{\exp \left(-\left[k_{2}+k_{1 R}\right] t\right)-\exp \left(-k_{5, b} t\right)}{k_{5, b}-k_{2}-k_{1 R}}-\frac{\exp \left(-\left[k_{3}+k_{4}\right] t\right)-\exp \left(-k_{5, b} t\right)}{k_{5, b}-k_{3}-k_{4}}\right\}
$$

and

$$
P_{C B, c}(t)=\frac{R_{C B, c} k_{3} k_{2} P_{0}}{k_{3}+k_{4}-\left\{k_{2}+k_{1 R}\right\}}\left\{\frac{\exp \left(-\left[k_{2}+k_{1 R}\right] t\right)-\exp \left(-k_{5, c} t\right)}{k_{5, c}-k_{2}-k_{1 R}}-\frac{\exp \left(-\left[k_{3}+k_{4}\right] t\right)-\exp \left(-k_{5, c} t\right)}{k_{5, c}-k_{3}-k_{4}}\right\} .
$$


Because of overall charge neutrality before and after photo-excitation, the HOMO hole population can be expressed through the electron populations above via

$$
P_{H}(\mathrm{t})=P_{L U M O}(\mathrm{t})+P_{I C T}(\mathrm{t})+P_{C B}(\mathrm{t})
$$

and approximated by

$$
P_{H}(\mathrm{t}) \cong P_{I C T}(\mathrm{t})+P_{C B}(\mathrm{t})
$$

since the LUMO population is depleted on timescales that are small compared to the instrument response function.

We emphasize that Fig. S9 illustrates all rates that could principally contribute to the interfacial dynamics, which is more than the number of free fit parameters used and, as outlined in the following, far more than the effective number of free fit parameters relevant for each individual section within the vast range of time-delays included in this study. In more detail: $\mathrm{k}_{1}$ indicates the initial photoexcitation, which occurs on attosecond timescales, $\mathrm{k}_{2}$ is the rate of dye oxidation, which is known to be completed within $<100 \mathrm{fs}$ (see e.g. Furube et al. ${ }^{46}$ ). These timescales are far below the temporal resolution of the experiment and, thus, are effectively zero for the purpose of this study. The intramolecular recombination rate $\mathrm{k}_{1 \mathrm{R}}$ is much smaller than the oxidation rate $\mathrm{k}_{2}$ and has no impact on the fit. In the fit procedure, the three rates $\mathrm{k}_{1}, \mathrm{k}_{2}$, and $\mathrm{k}_{1 \mathrm{R}}$ (marked gray in Fig. S9) are fixed to literature values. Since the ICT-to-CB injection rate $\mathrm{k}_{3}$ is much higher than the recombination rates $\mathrm{k}_{4}$ and $\mathrm{k}_{5}$ (Fig. S9(a)), the data in Fig. 2(a) reach a plateau at $\sim 1$ ns delay and $\mathrm{k}_{3}$ is effectively the only free fit parameter to describe the early dynamics in Fig. 2(a) for pumpprobe delays below $3 \mathrm{~ns}$. Conversely, none of the parameters $\mathrm{k}_{i}$ with $\mathrm{i}=1 \ldots 4$ have any impact on the slow recombination dynamics at longer delays $>3 \mathrm{~ns}$. These are entirely governed by the three recombination rates $\mathrm{k}_{5, \mathrm{~b}, \mathrm{c}}$ (Fig. S9(b)), which are readily apparent from the 3 different decay slopes for delays $\geq 4 \mathrm{~ns}$ in Fig. 2(a). The range of these timescales ( $\sim 10 \mathrm{~ns}, \sim 100 \mathrm{~ns}, \sim 2 \mu \mathrm{s})$ is in reasonable agreement with the results of Bauer et al., ${ }^{9}$ who report recombination time scales of 290 ns and 2.6 $\mu \mathrm{s}$. In brief, the number of effective free fit parameters is reduced to 1 for short time delays ( $\leq 2 \mathrm{~ns}$ ) and 3 for long time delays ( $\geq 3 \mathrm{~ns}$ ), whereby each of these contributions are readily apparent from the data in Fig. 2(a): $\mathrm{k}_{3}$ from the delayed onset of the $\mathrm{Zn} 3 \mathrm{~d}$ compared to the $\mathrm{C} 1 \mathrm{~s}$ response, and $\mathrm{k}_{5 \mathrm{a}, \mathrm{b}, \mathrm{c}}$ from the 3 decay slopes in the logarithmic section of the figure. We therefore trust that the parameter values derived from the fits are meaningful and well-defined. We note that we also tested alternative fit models, such as a stretched exponential and a single-channel sequential recombination, whereby the three observed recombination timescales would be associated with three sequential steps of a single recombination channel. None of the alternatives described the 
data as well as the model of three independent recombination channels illustrated in Fig. S9(b). This finding is consistent with a picture of (at least) three different recombination channels that may be associated, for example, with different levels occupied by the injected electrons in the substrate. The currently available data, however, do not provide access to this level of detail.

\section{I) Time-resolved XPS of N3 adsorbed to a gold substrate}

The difference $\Delta \mathrm{E}_{\mathrm{N} 3}$ between the transient energy shifts of the $\mathrm{C} 1 \mathrm{~s} / \mathrm{Ru} 3 \mathrm{~d}$ photolines and the surface band bending is interpreted as an interfacial potential drop between the molecular HOMO hole and the semiconductor surface, as described in section $\mathrm{G}$ and in the main manuscript. This interpretation is supported by trXPS data of an N3 film drop-cast onto a thin film of gold. The experimental setup used for these measurements is identical to one described in section A. For the N3/Au sample, perfect screening of injected electrons and the absence of any band bending effects in the metal substrate are expected to reduce the photoresponse of the entire sample to the pure molecular response $\Delta \mathrm{E}_{\mathrm{N} 3}$. Figure $\mathrm{S} 10$ (a) shows the $\mathrm{C} 1 \mathrm{~s} / \mathrm{Ru} 3 \mathrm{~d}$ response of the $\mathrm{N} 3 / \mathrm{Au}$ sample at a pump-probe delay of $\Delta \mathrm{t}=2 \mathrm{~ns}$ (red) compared to the GS spectrum (blue). Solid lines are the result of the fit procedure described in section B. Subtracting a rigidly $(148 \mathrm{meV})$ shifted GS spectrum from the ES spectrum results in a residual with no discernable structure beyond the signal-to-noise level (Fig. S10(b)), as observed for the $\mathrm{N} 3 / \mathrm{ZnO}$ system (Fig. 1(c)). Figure S10(c) shows the temporal evolution of the photoinduced spectral shift. In particular, the inset illustrates the onset of this shift within the instrument response ( $\sim 70 \mathrm{ps}$ ). This is in contrast to the $\mathrm{C} 1 \mathrm{~s} / \mathrm{Ru} 3 \mathrm{~d}$ response of the $\mathrm{N} 3 / \mathrm{ZnO}$ system, where an additional, slower band bending contribution is present in the overall onset of the $\mathrm{C} 1 \mathrm{~s} / \mathrm{Ru} 3 \mathrm{~d}$ response (Fig. 2(a)). The IRF-limited response for the N3/Au system supports the interpretation of $\Delta \mathrm{E}_{\mathrm{N} 3}$ as a purely molecular response leading to a potential difference between the molecule itself and the substrate surface. The data shown in Fig. S10(d) verify that the $\mathrm{N} 3 / \mathrm{Au}$ photo-response is indeed due to photoexcitation of the N3 dye. At a relatively high laser fluence of $5 \mathrm{~mJ} / \mathrm{cm}^{2}$ and a low scanning speed of $100 \mu \mathrm{m} / \mathrm{s}$, the dye molecules are gradually and irreversibly damaged, as evidenced by the decreasing amplitude of the $C 1 \mathrm{~s} / \mathrm{Ru3d} \operatorname{shift}(\Delta t=100 \mathrm{ps})$ in subsequent scans on the same sample area. This provides strong evidence that the photo-induced dynamics and associated transient trXPS responses are due to light-harvesting by intact N3 chromophores. 

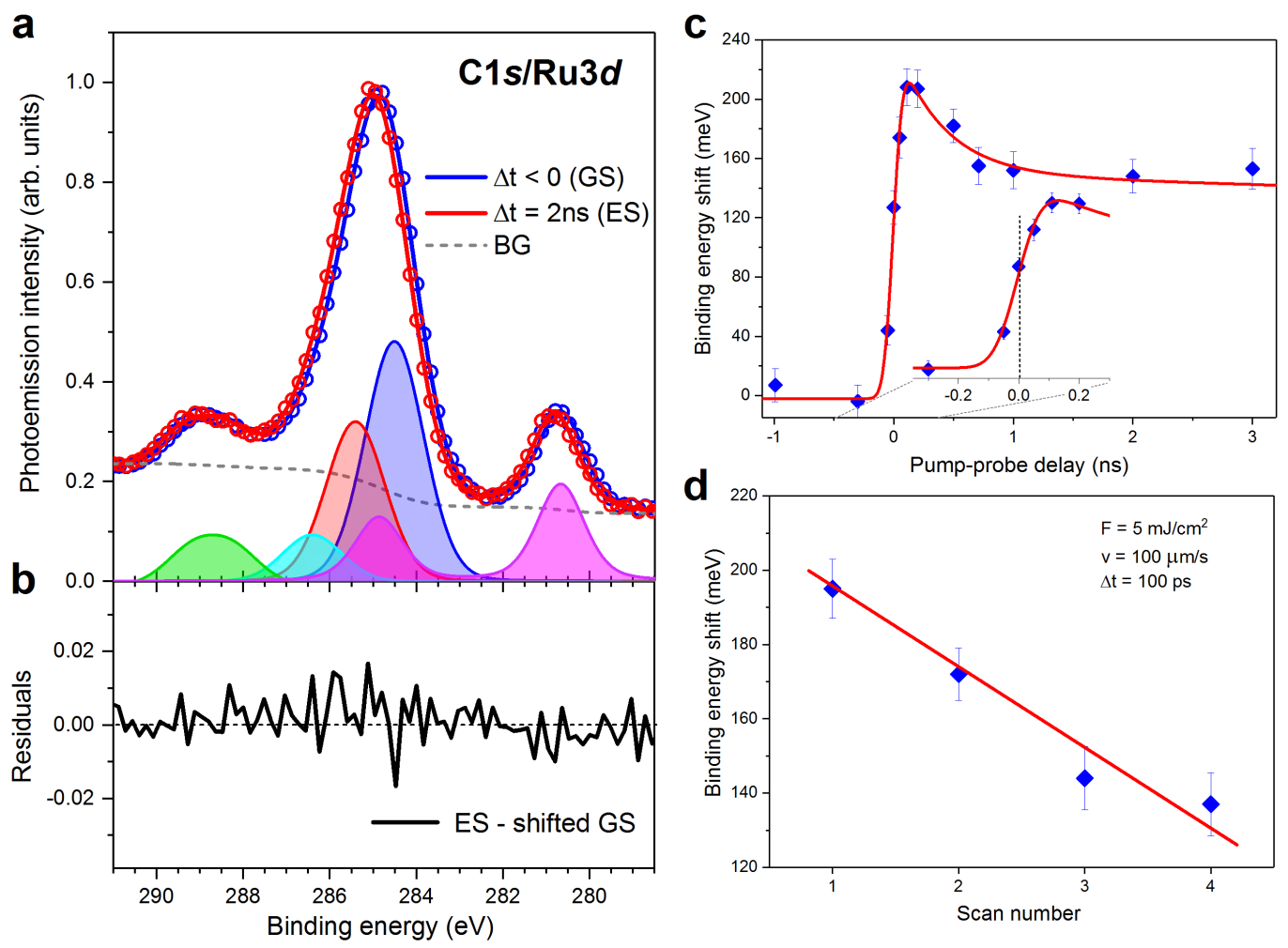

Figure S10. Photoresponse of the $\mathrm{C} 1 \mathrm{~s} / \mathrm{Ru} 3 \mathrm{~d}$ lines of $\mathrm{N} 3$ adsorbed to a gold substrate. a GS (blue) and ES (red) trXPS data (circles) and fits (lines) for the N3/Au system at 2 ns pumpprobe delay. b Residual trace after modelling the photo-induced effect by a rigid shift of $148 \mathrm{meV}$. c Transient shifts within the first three nanoseconds after photoexcitation. d Sample degradation becomes apparent through diminishing maximum binding energy shifts of the $\mathrm{C} 1 \mathrm{~s} / \mathrm{Ru} 3 \mathrm{~d}$ photoline when scanning over the same sample area multiple times. 


\section{References}

(1) Bauer, C.; Boschloo, G.; Mukhtar, E.; Hagfeldt, A. Ultrafast Relaxation Dynamics of Charge Carriers Relaxation in ZnO Nanocrystalline Thin Films. Chem. Phys. Lett. 2004, 387, 176-181.

(2) Makuła, P.; Pacia, M.; Macyk, W. How To Correctly Determine the Band Gap Energy of Modified Semiconductor Photocatalysts Based on UV-Vis Spectra. J. Phys. Chem. Lett. 2018, 9, 6814-6817.

(3) Tauc, J.; Grigorovici, R.; Vancu, A. Optical Properties and Electronic Structure of Amorphous Germanium. Phys. Status Solidi B 1966, 15, 627-637.

(4) Özgür, Ü.; Alivov, Ya. I.; Liu, C.; Teke, A.; Reshchikov, M. A.; Doğan, S.; Avrutin, V.; Cho, S.-J.; Morkoç, H. A Comprehensive Review of ZnO Materials and Devices. J. Appl. Phys. 2005, 98, 041301.

(5) Chou, T. P.; Zhang, Q.; Cao, G. Effects of Dye Loading Conditions on the Energy Conversion Efficiency of $\mathrm{ZnO}$ and $\mathrm{TiO}_{2}$ Dye-Sensitized Solar Cells. J. Phys. Chem. C 2007, $111,18804-18811$.

(6) Horiuchi, H.; Katoh, R.; Hara, K.; Yanagida, M.; Murata, S.; Arakawa, H.; Tachiya, M. Electron Injection Efficiency from Excited N3 into Nanocrystalline ZnO Films: Effect of (N3-Zn ${ }^{2+}$ ) Aggregate Formation. J. Phys. Chem. B 2003, 107, 2570-2574.

(7) Keis, K.; Bauer, C.; Boschloo, G.; Hagfeldt, A.; Westermark, K.; Rensmo, H.; Siegbahn, H. Nanostructured ZnO Electrodes for Dye-Sensitized Solar Cell Applications. J. Photochem. Photobiol. Chem. 2002, 148, 57-64.

(8) Neppl, S.; Shavorskiy, A.; Zegkinoglou, I.; Fraund, M.; Slaughter, D. S.; Troy, T.; Ziemkiewicz, M. P.; Ahmed, M.; Gul, S.; Rude, B.; Zhang, J. Z.; Tremsin, A. S.; Glans, P.A.; Liu, Y.-S.; Wu, C. H.; Guo, J.; Salmeron, M.; Bluhm, H.; Gessner, O. Capturing Interfacial Photoelectrochemical Dynamics with Picosecond Time-Resolved X-Ray Photoelectron Spectroscopy. Faraday Discuss. 2014, 171, 219-241.

(9) Bauer, C.; Boschloo, G.; Mukhtar, E.; Hagfeldt, A. Electron Injection and Recombination in $\mathrm{Ru}(\mathrm{Dcbpy})_{2}(\mathrm{NCS})_{2}$ Sensitized Nanostructured ZnO. J. Phys. Chem. B 2001, 105, 55855588.

(10) Ozawa, K.; Emori, M.; Yamamoto, S.; Yukawa, R.; Yamamoto, S.; Hobara, R.; Fujikawa, K.; Sakama, H.; Matsuda, I. Electron-Hole Recombination Time at $\mathrm{TiO}_{2}$ Single-Crystal Surfaces: Influence of Surface Band Bending. J. Phys. Chem. Lett. 2014, 5, 1953-1957.

(11) Thompson, A.; Lindau, I.; Attwood, D.; Liu, Y.; Gullikson, E.; Pianetta, P.; Howells, M.; Robinson, A.; Kim, K.-J.; Scofield, J.; Kirz, J.; Underwood, J.; Kortright, J.; Williams, G.; Winick, H. X-Ray Data Booklet; 3rd ed.; https://xdb.lbl.gov/xdb-new.pdf; Berkeley, CA, USA, 2009.

(12) Mayor, L. C.; Saywell, A.; Magnano, G.; Satterley, C. J.; Schnadt, J.; O’Shea, J. N. Adsorption of a Ru(II) Dye Complex on the Au(111) Surface: Photoemission and Scanning Tunneling Microscopy. J. Chem. Phys. 2009, 130, 164704.

(13) King, P. D. C.; Veal, T. D.; Schleife, A.; Zúñiga-Pérez, J.; Martel, B.; Jefferson, P. H.; Fuchs, F.; Muñoz-Sanjosé, V.; Bechstedt, F.; McConville, C. F. Valence-Band Electronic Structure of $\mathrm{CdO}, \mathrm{ZnO}$, and $\mathrm{MgO}$ from x-Ray Photoemission Spectroscopy and QuasiParticle-Corrected Density-Functional Theory Calculations. Phys. Rev. B 2009, 79, 205205.

(14) Mahl, J.; Gessner, O.; Barth, J. V.; Feulner, P.; Neppl, S. Strong Potential Gradients and Electron Confinement in $\mathrm{ZnO}$ Nanoparticle Films: Implications for Charge-Carrier Transport and Photocatalysis. ACS Appl. Nano Mater. 2021, 4, 12213-12221. 
(15) Zhang, Z.; Yates, J. T. Band Bending in Semiconductors: Chemical and Physical Consequences at Surfaces and Interfaces. Chem. Rev. 2012, 112, 5520-5551.

(16) Zojer, E.; Taucher, T. C.; Hofmann, O. T. The Impact of Dipolar Layers on the Electronic Properties of Organic/Inorganic Hybrid Interfaces. Adv. Mater. Interfaces 2019, 6, 1900581.

(17) Stähler, J.; Rinke, P. Global and Local Aspects of the Surface Potential Landscape for Energy Level Alignment at Organic-ZnO Interfaces. Chem. Phys. 2017, 485-486, 149-165.

(18) Gleason-Rohrer, D. C.; Brunschwig, B. S.; Lewis, N. S. Measurement of the Band Bending and Surface Dipole at Chemically Functionalized Si(111)/Vacuum Interfaces. J. Phys. Chem. C 2013, 117, 18031-18042.

(19) Favaro, M.; Jeong, B.; Ross, P. N.; Yano, J.; Hussain, Z.; Liu, Z.; Crumlin, E. J. Unravelling the Electrochemical Double Layer by Direct Probing of the Solid/Liquid Interface. Nat. Commun. 2016, 7, 1-8.

(20) Lichterman, M. F.; Hu, S.; Richter, M. H.; Crumlin, E. J.; Axnanda, S.; Favaro, M.; Drisdell, W.; Hussain, Z.; Mayer, T.; Brunschwig, B. S.; Lewis, N. S.; Liu, Z.; Lewerenz, H.-J. Direct Observation of the Energetics at a Semiconductor/Liquid Junction by Operando X-Ray Photoelectron Spectroscopy. Energy Environ. Sci. 2015, 8, 2409-2416.

(21) Minohara, M.; Horiba, K.; Kumigashira, H.; Ikenaga, E.; Oshima, M. Depth Profiling the Potential in Perovskite Oxide Heterojunctions Using Photoemission Spectroscopy. Phys. Rev. B 2012, 85, 165108.

(22) Sushko, P. V.; Chambers, S. A. Extracting Band Edge Profiles at Semiconductor Heterostructures from Hard-x-Ray Core-Level Photoelectron Spectra. Sci. Rep. 2020, 10, 13028.

(23) Wippler, D.; Wilks, R. G.; Pieters, B. E.; van Albada, S. J.; Gerlach, D.; Hüpkes, J.; Bär, M.; Rau, U. Pronounced Surface Band Bending of Thin-Film Silicon Revealed by Modeling Core Levels Probed with Hard X-Rays. ACS Appl. Mater. Interfaces 2016, 8, 17685-17693.

(24) Tsuchiya, T.; Itoh, Y.; Yamaoka, Y.; Ueda, S.; Kaneko, Y.; Hirasawa, T.; Suzuki, M.; Terabe, K. In Situ Hard X-Ray Photoelectron Spectroscopy of Space Charge Layer in a ZnO-Based All-Solid-State Electric Double-Layer Transistor. J. Phys. Chem. C 2019, 123, 10487-10493.

(25) Shavorskiy, A.; Ye, X.; Karslıoğlu, O.; Poletayev, A. D.; Hartl, M.; Zegkinoglou, I.; Trotochaud, L.; Nemšák, S.; Schneider, C. M.; Crumlin, E. J.; Axnanda, S.; Liu, Z.; Ross, P. N.; Chueh, W.; Bluhm, H. Direct Mapping of Band Positions in Doped and Undoped Hematite during Photoelectrochemical Water Splitting. J. Phys. Chem. Lett. 2017, 8, 55795586.

(26) Tanuma, S.; Powell, C. J.; Penn, D. R. Calculations of Electron Inelastic Mean Free Paths. V. Data for 14 Organic Compounds over the 50-2000 EV Range. Surf. Interface Anal. 1994, $21,165-176$.

(27) Morkoç, H.; Özgür, Ü. Zinc Oxide: Fundamentals, Materials and Device Technology; 2009; Vol. Weinheim, Germany: Wiley-VCH.

(28) Cahen, D.; Kahn, A. Electron Energetics at Surfaces and Interfaces: Concepts and Experiments. Adv. Mater. 2003, 15, 271-277.

(29) Taucher, T. C.; Hehn, I.; Hofmann, O. T.; Zharnikov, M.; Zojer, E. Understanding Chemical versus Electrostatic Shifts in X-Ray Photoelectron Spectra of Organic Self-Assembled Monolayers. J. Phys. Chem. C 2016, 120, 3428-3437.

(30) Lueth, H. Solid Surfaces, Interfaces and Thin Films; 5th ed.; pp. 527-528; Springer: Berlin Heidelberg, 2010. 
(31) Miyashita, M.; Sunahara, K.; Nishikawa, T.; Uemura, Y.; Koumura, N.; Hara, K.; Mori, A.; Abe, T.; Suzuki, E.; Mori, S. Interfacial Electron-Transfer Kinetics in Metal-Free Organic Dye-Sensitized Solar Cells: Combined Effects of Molecular Structure of Dyes and Electrolytes. J. Am. Chem. Soc. 2008, 130, 17874-17881.

(32) Kavan, L.; Grätzel, M.; Gilbert, S. E.; Klemenz, C.; Scheel, H. J. Electrochemical and Photoelectrochemical Investigation of Single-Crystal Anatase. J. Am. Chem. Soc. 1996, 118, 6716-6723.

(33) Shklover, V.; Nazeeruddin, M.-K.; Zakeeruddin, S. M.; Barbé, C.; Kay, A.; Haibach, T.; Steurer, W.; Hermann, R.; Nissen, H.-U.; Grätzel, M. Structure of Nanocrystalline $\mathrm{TiO}_{2}$ Powders and Precursor to Their Highly Efficient Photosensitizer. Chem. Mater. 1997, 9, 430-439.

(34) Yoshihara, T.; Katoh, R.; Furube, A.; Murai, M.; Tamaki, Y.; Hara, K.; Murata, S.; Arakawa, H.; Tachiya, M. Quantitative Estimation of the Efficiency of Electron Injection from Excited Sensitizer Dye into Nanocrystalline ZnO Film. J. Phys. Chem. B 2004, 108, 2643-2647.

(35) Grätzel, M. Solar Energy Conversion by Dye-Sensitized Photovoltaic Cells. Inorg. Chem. 2005, 44, 6841-6851.

(36) Bastide, S.; Butruille, R.; Cahen, D.; Dutta, A.; Libman, J.; Shanzer, A.; Sun, L.; Vilan, A. Controlling the Work Function of GaAs by Chemisorption of Benzoic Acid Derivatives. $J$. Phys. Chem. B 1997, 101, 2678-2684.

(37) Rühle, S.; Greenshtein, M.; Chen, S.-G.; Merson, A.; Pizem, H.; Sukenik, C. S.; Cahen, D.; Zaban, A. Molecular Adjustment of the Electronic Properties of Nanoporous Electrodes in Dye-Sensitized Solar Cells. J. Phys. Chem. B 2005, 109, 18907-18913.

(38) Vilan, A.; Ghabboun, J.; Cahen, D. Molecule-Metal Polarization at Rectifying GaAs Interfaces. J. Phys. Chem. B 2003, 107, 6360-6376.

(39) Bruening, M.; Cohen, R.; Guillemoles, J. F.; Moav, T.; Libman, J.; Shanzer, A.; Cahen, D. Simultaneous Control of Surface Potential and Wetting of Solids with Chemisorbed Multifunctional Ligands. J. Am. Chem. Soc. 1997, 119, 5720-5728.

(40) Salomon, A.; Berkovich, D.; Cahen, D. Molecular Modification of an Ionic SemiconductorMetal Interface: ZnO/Molecule/Au Diodes. Appl. Phys. Lett. 2003, 82, 1051-1053.

(41) Chen, H.; Cole, J. M.; Stenning, G. B. G.; Yanguas-Gil, A.; Elam, J. W.; Stan, L.; Gong, Y. Imaging Dye Aggregation in MK-2, N3, N749, and SQ-2 Dye $\cdots \mathrm{TiO}_{2}$ Interfaces That Represent Dye-Sensitized Solar Cell Working Electrodes. ACS Appl. Energy Mater. 2020, 3, 3230-3241.

(42) Siefermann, K. R.; Pemmaraju, C. D.; Neppl, S.; Shavorskiy, A.; Cordones, A. A.; VuraWeis, J.; Slaughter, D. S.; Sturm, F. P.; Weise, F.; Bluhm, H.; Strader, M. L.; Cho, H.; Lin, M.-F.; Bacellar, C.; Khurmi, C.; Guo, J.; Coslovich, G.; Robinson, J. S.; Kaindl, R. A.; Schoenlein, R. W.; Belkacem, A.; Neumark, D. M.; Leone, S. R.; Nordlund, D.; Ogasawara, H.; Krupin, O.; Turner, J. J.; Schlotter, W. F.; Holmes, M. R.; Messerschmidt, M.; Minitti, M. P.; Gul, S.; Zhang, J. Z.; Huse, N.; Prendergast, D.; Gessner, O. Atomic-Scale Perspective of Ultrafast Charge Transfer at a Dye-Semiconductor Interface. J. Phys. Chem. Lett. 2014, 5, 2753-2759.

(43) Oh, D. H.; Sano, M.; Boxer, S. G. Electroabsorption (Stark Effect) Spectroscopy of Monoand Biruthenium Charge-Transfer Complexes: Measurements of Changes in Dipole Moments and Other Electrooptic Properties. J. Am. Chem. Soc. 1991, 113, 6880-6890. 
(44) Sampaio, R. N.; Li, G.; Meyer, G. J. Continuous Surface Electric Field Contraction Accompanying Electron Transfer from $\mathrm{TiO}_{2}$ to Oxidized Sensitizers. ACS Energy Lett. 2016, 1, 846-851.

(45) Ardo, S.; Sun, Y.; Staniszewski, A.; Castellano, F. N.; Meyer, G. J. Stark Effects after Excited-State Interfacial Electron Transfer at Sensitized $\mathrm{TiO}_{2}$ Nanocrystallites. J. Am. Chem. Soc. 2010, 132, 6696-6709.

(46) Furube, A.; Katoh, R.; Hara, K.; Murata, S.; Arakawa, H.; Tachiya, M. Ultrafast Stepwise Electron Injection from Photoexcited Ru-Complex into Nanocrystalline ZnO Film via Intermediates at the Surface. J. Phys. Chem. B 2003, 107, 4162-4166. 